\title{
Hayabusa2's superior solar conjunction mission operations: Planning and post-operation results
}

\author{
Stefania Soldini ${ }^{1,2}(\bowtie)$, Hiroshi Takeuchi ${ }^{2}$, Sho Taniguchi ${ }^{3}$, Shota Kikuchi ${ }^{2}$, Yuto Takei ${ }^{2}$, Go \\ Ono $^{2}$, Masaya Nakano ${ }^{3}$, Takafumi Ohnishi ${ }^{3}$, Takanao Saiki ${ }^{2}$, Yuichi Tsuda ${ }^{2}$, Fuyuto Terui ${ }^{2}$, \\ Naoko Ogawa ${ }^{2}$, Yuya Mimasu ${ }^{2}$, Tadateru Takahashi ${ }^{2}$, Atsushi Fujii ${ }^{2}$, Satoru Nakazawa $^{2}$, \\ Kent Yoshikawa ${ }^{2}$, Yusuke Oki ${ }^{2}$, Chikako Hirose ${ }^{2}$, Hirotaka Sawada ${ }^{2}$, Tomohiro Yamaguchi ${ }^{2,4}$, \\ and Makoto Yoshikawa ${ }^{2}$ \\ 1. Department of Mechanical, Materials and Aerospace Engineering, University of Liverpool, Liverpool L69 3BX, UK \\ 2. Institute of Space and Astronautical Science, Japan Aerospace Exploration Agency, Sagamihara 252-5210, Japan \\ 3. Fujitsu Limited, Tokyo 105-7123, Japan \\ 4. Mitsubishi Electric Corporation, Tokyo 100-8310, Japan
}

\begin{abstract}
In late 2018, the asteroid Ryugu was in the Sun's shadow during the superior solar conjunction phase. As the Sun-Earth-Ryugu angle decreased to below $3^{\circ}$, the Hayabusa 2 spacecraft experienced 21 days of planned blackout in the Earth-probe communication link. This was the first time a spacecraft had experienced solar conjunction while hovering around a minor body. For the safety of the spacecraft, a low energy transfer trajectory named Ayu was designed in the Hill reference frame to increase its altitude from 20 to $110 \mathrm{~km}$. The trajectory was planned with the newly developed optNEAR tool and validated with real time data. This article shows the results of the conjunction operation, from planning to flight data.

\section{ABSTRACT}

\section{KEYWORDS}

superior solar conjunction

Hayabusa2

Ryugu

hovering satellite

mission operations

Research Article

Received: 18 Januany 2020

Accepted: 26 March 2020

(C) The Author(s) 2020

\section{Introduction}

The Hayabusa2 mission was a Japanese robotic mission to Ryugu [1]. Since rendezvousing with Ryugu less than one year ago, Hayabusa2 has set a new first for Japan by successfully performing the first ever impact experiment on an asteroid (April 2019). The impact experiment was executed after successful completion of another critical operation: the touchdown operation for sampling Ryugu's surface (February 2018). The first touchdown was followed by a second successful touchdown at the location of the small carry-on impactor's (SCI) artificial crater site in July 2019. After entering the Sun's shadow in late 2018 with the start of the superior solar conjunction phase, Hayabusa2 successfully deployed two rovers (September 2018) and a lander (October 2018). In November 2019, Hayabusa2 completed its exploration phase and began its return journey towards the Earth.
Contrary to NASA's OSIRIS-REx mission [2], the Hayabusa2 spacecraft did not orbit Ryugu, but instead hovered at a relative distance of $20 \mathrm{~km}$ from its center, known as the home position (HP) point [3]. Navigation was performed in the HP frame, with the $z$-axis aligned with the asteroid-Earth line. Hayabusa2 typically operates at around $20 \mathrm{~km}$ altitude in $+z_{\mathrm{HP}}$, known as controlled BOX-A [3]. To maintain Hayabusa2's position in BOX-A, a $\Delta V$ command was sent to the spacecraft every 1-2 days. A decrease in the Sun-Earth-probe (SEP) angle below $3^{\circ}$ caused a substantial increase in data noise in the Doppler measurements [4], thus making it difficult to correctly send commands to the spacecraft. JAXA's previous Hayabusa mission experienced solar conjunction during the transfer phase [5], when it was placed in a heliocentric orbit towards Itokawa. It was the first time that a spacecraft experienced superior solar conjunction 
Nomenclature

\begin{tabular}{|c|c|}
\hline AIT & asteroid image tracking \\
\hline $\mathrm{AOCS}$ & attitude orbit control system \\
\hline $\mathrm{AU}$ & astronomic unit \\
\hline COI & conjunction orbit insertion \\
\hline FD & flight dynamics \\
\hline FOV & field of view \\
\hline GCP-NAV & ground control point navigation \\
\hline goNEAR & gravitational orbits near earth asteroid regions \\
\hline HGA & high gain antenna \\
\hline $\mathrm{HP}$ & home position ( $20 \mathrm{~km}$ from Ryugu) \\
\hline HPNAV & home position NAVigation \\
\hline HRM & home position recovery maneuver \\
\hline JATOPS & JAXA approach trajectory optimizer with stochastic constraints \\
\hline OD & orbit determination \\
\hline optNEAR & optimum trajectory near Earth asteroid regions \\
\hline $\mathrm{RCS}$ & reaction control system \\
\hline SEP & Sun-Earth-probe \\
\hline SRP & solar radiation pressure \\
\hline TCM & trajectory control maneuver \\
\hline ToF & time of flight \\
\hline UTC & universal coordinated time \\
\hline
\end{tabular}

while in the hovering phase. This condition lasted 21 days for Hayabusa2, making the standard 1-2 days HP maintenance operation infeasible. As a $20 \mathrm{~km}$ altitude is usually artificially maintained, it was too risky to leave the spacecraft uncontrolled in proximity to Ryugu. To prevent a close approach with the asteroid, or an undesired escape from Ryugu's sphere of influence, the optimum trajectory near-Earth asteroid regions (optNEAR) tool was developed for the design of a low energy transfer trajectory for hovering satellites. The trajectory was designed in the Hill frame and due to its fish-like shape was named "ayu" (Japanese sweetfish) trajectory $[6,7]$. For the case of Hayabusa2, the ayu trajectory was designed to reach an altitude of $110 \mathrm{~km}$ in deep conjunction (minimum SEP angle). Only two deterministic maneuvers were required, with a $\Delta V$ budget of less than $1 \mathrm{~m} / \mathrm{s}$. The shooting method developed in optNEAR takes advantage of the natural dynamics of the asteroid-Sun system, knowing that in the Hill problem the spacecraft motion is opposed by the solar radiation pressure (SRP) acceleration, for a fixed initial energy level. This principle was previously used by JAXA's Hiten mission [8], for the design of a recovery trajectory in the patched Sun-Earth and Earth-Moon systems [9]. The ayu trajectory aimed to direct the spacecraft towards the zero velocity curves of the Hill problem (boundary of possible motion), where the maximum altitude of $110 \mathrm{~km}$ was reached and the return to a $20 \mathrm{~km}$ altitude could therefore be executed in fuel- free mode (ballistic capture). As a first approximation, the conjunction trajectory was designed in the Hill frame of the Sun-asteroid system and the solution was then refined in the full-ephemeris problem $[6,7]$. The time of flight (ToF) of the flown ayu conjunction trajectory was around 38 days, with two deterministic $\Delta V$ designed at the conjunction orbit insertion (COI) point (home position (HP) before the conjunction) and at the home position recovery (HRM) point (HP after the conjunction). Two trajectory correction maneuvers (TCMs) were scheduled before and after the deep conjunction phase. The trajectory designed with the optNEAR tool was validated in real time operations and used for testing the JAXA's trajectory design JATOPS (JAXA approach trajectory optimizer with stochastic constraints) tool for high altitude operations. The results of the post-flight operations are presented here.

\section{Solar conjunction mission design and operation planning}

The ayu conjunction trajectory was designed in the Hill reference frame, as shown in Soldini et al. [6,7]. The dynamics of the mother spacecraft was written in a rotating reference frame, where the system was centered on Ryugu and the Sun, and the asteroid was placed along the $x$-axis. The Sun was in the negative $x$ coordinates. Depending on how the initial energy of 
the spacecraft (state vector) was set, it was possible to distinguish regions of motion where the spacecraft dynamics was not permitted [10]. This information was used to increase the spacecraft's altitude from $20 \mathrm{~km}$ to a safety altitude during deep conjunction. On 2018/12/11, the spacecraft reached the deep conjunction position located at the boundary of the permitted motion, as seen in the Hill reference frame. In deep conjunction, the SEP angle was at its minimum value of $0.4^{\circ}$.

The conjunction operation started and ended when the SEP angle was equal to $5^{\circ}$ and the gravity constant of Ryugu, $\mu_{a}$, was set to $30 \mathrm{~m}^{3} / \mathrm{s}^{2}$, as in Soldini et al. $[6,7]$. Figure 1 shows the nominal conjunction trajectory as seen from the Hill reference frame and HP reference frame (Figs. 1(a) and 1(b)). The trajectory's fish-like shape in the Hill coordinates can be seen in Fig. 1(a). The $z$-axis of the HP reference frame was along the Earth-asteroid line pointing towards the Earth. The Sun-asteroid line belonged to the positive coordinates of the $x-z$ plane and the $y$ axis was given such that the HP frame was a right-handed coordinate system. Figure 1(b) shows that the ayu trajectory was a periodic orbit when placed in the HP reference frame. Indeed, COI and HRM share the same coordinates in this frame. Figure 1 also shows the epochs of the TCMs (red points) and the deep conjunction epoch (green point).

The ayu conjunction trajectory requires two deterministic maneuvers: before and after the superior solar conjunction at the COI point and the HRM, respectively (Fig. 1) [6,7]. The total contribution of the two deterministic $\Delta V$ maneuvers at COI and HRM computed with the optNEAR tool was $0.2359 \mathrm{~m} / \mathrm{s}$

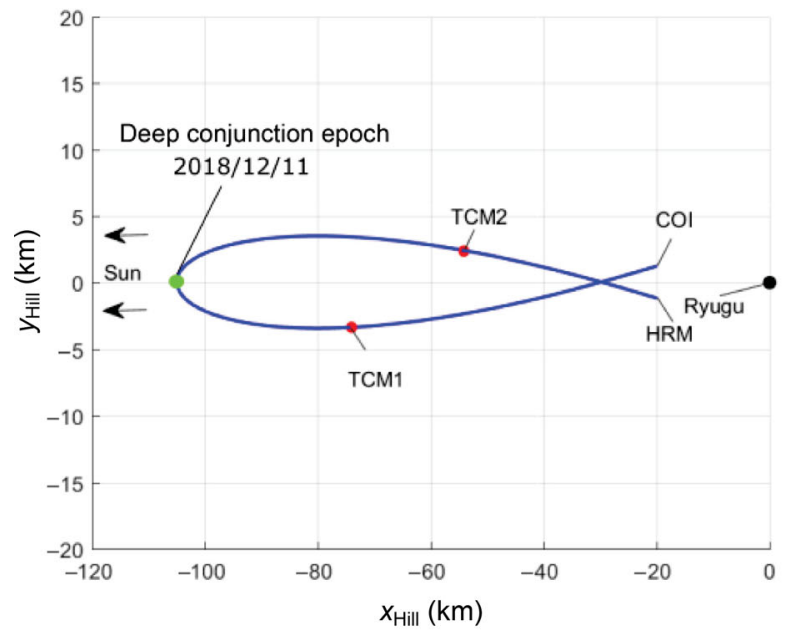

(a) Hill reference frame
$[6,7]$. As a result of an uncertainty analysis in the deterministic maneuvers at COI and HRM, Soldini et al. concluded that at least two stochastic TCMs were required $[6,7]$, as shown by the red point in Fig. 1 . The conjunction operation required four maneuvers to be performed. The solution in the Hill reference frame was the first guess solution. The trajectory was then refined and recomputed in the full ephemeries planetary equations via the use of NASA's SPICE toolkit, interfaced with the optNEAR tool.

Table 1 shows the epochs of the Hayabusa2's superior solar conjunction operations as a function of the SEP angle. The overall solar conjunction phase lasted for 37 days and for 21 days the spacecraft was kept free from on ground control, while in deep conjunction. Note that on $2018 / 12 / 28$, it was decided to modify the $\Delta V$ planning at HRM (last line in Table 1) and the home position keeping (HPK) maneuver for hovering position maintenance (20 km from Ryugu along the $z_{\mathrm{HP}}$ axis) was merged with the HRM maneuver.

In Soldini et al. [6,7], it was demonstrated that the ayu conjunction trajectory allowed a low fuel expenditure and Ryugu was always in the field of view (FOV) of Hayabusa2's wide angle navigation camera

Table 1 Scheduled maneuvers for the Hayabusa2's superior solar conjunction

\begin{tabular}{lcc}
\hline Maneuver & Epoch $($ UTC) & SEP angle $\left(^{\circ}\right)$ \\
\hline COI & $2018 / 11 / 23$ & 5 \\
TCM1 & $2018 / 11 / 30$ & 3 \\
TCM2 & $2018 / 12 / 25$ & 4 \\
HRM+HPK & $2018 / 12 / 29$ & 5 \\
\hline
\end{tabular}

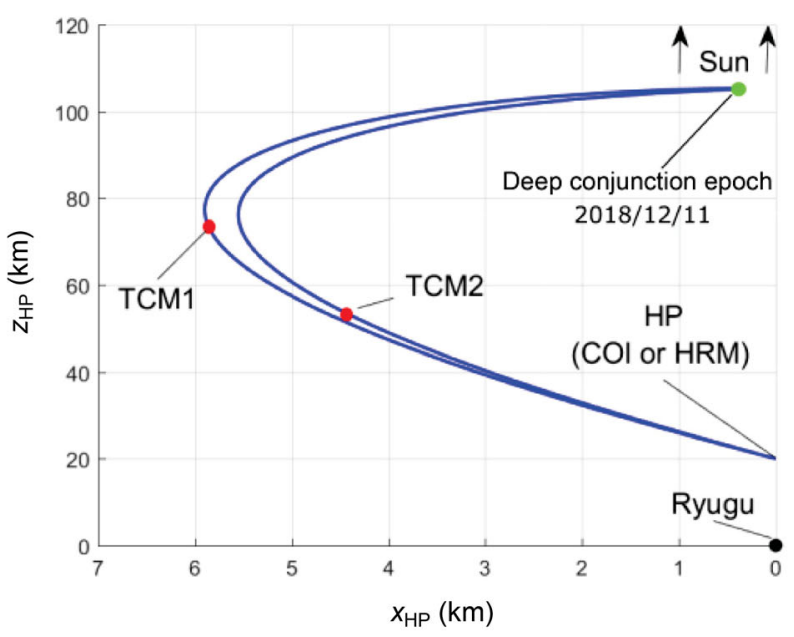

(b) HP reference frame

Fig. 1 Design of the solar conjunction ayu trajectory as seen in the Hill reference frame (a) and HP reference frame (b). 
ONC-W1 $\left(60^{\circ}\right)$. Figure 2 shows the ayu trajectory for $\mu_{a}=32 \mathrm{~m}^{3} / \mathrm{s}^{2}$ and for a conjunction maneuver starting at a SEP angle of $6^{\circ}$. Figure 2(a) shows the trajectory by forward (black) and backward (green) integration from the deep conjunction point $(-H)$, as shown by the green point in Fig. 1. The geometry of the camera was verified when the spacecraft was kept Earthpointing ( $\sim$ Sun-pointing in deep conjunction). Figure 2 (b) shows the angle between the $x$-axis direction and the spacecraft-Ryugu line (half of the camera FOV). The asteroid was always in the FOV of the ONC-W1 camera and in some cases was within the ONC-T camera FOV $\left(6^{\circ}\right)$.

For the solar conjunction mission planning, four main phases were defined and the epoch of the maneuvers are given in Table 1:

(1) Preparation phase: COI (2018/11/23)-TCM1 (2018/11/30). During the preparation phase, the spacecraft performed a $180^{\circ}$ slew maneuver around the $z_{\mathrm{HP}}$-axis to ensure the correct orientation of the 12 thrusters after the deep conjunction phase (flip of the HP frame). The COI maneuver was performed when the SEP angle was $5^{\circ}$ and TCM1 was performed when the SEP angle was $3^{\circ}$.

(2) Deep conjunction phase: TCM1 (2018/12/01)TCM2 (2018/12/21). When the spacecraft was in deep conjunction (SEP angle $<3^{\circ}$ ), the spacecraft did not perform any orbit maneuvers, only attitude maintenance. Beacon operations were carried out to monitor the status of the spacecraft while

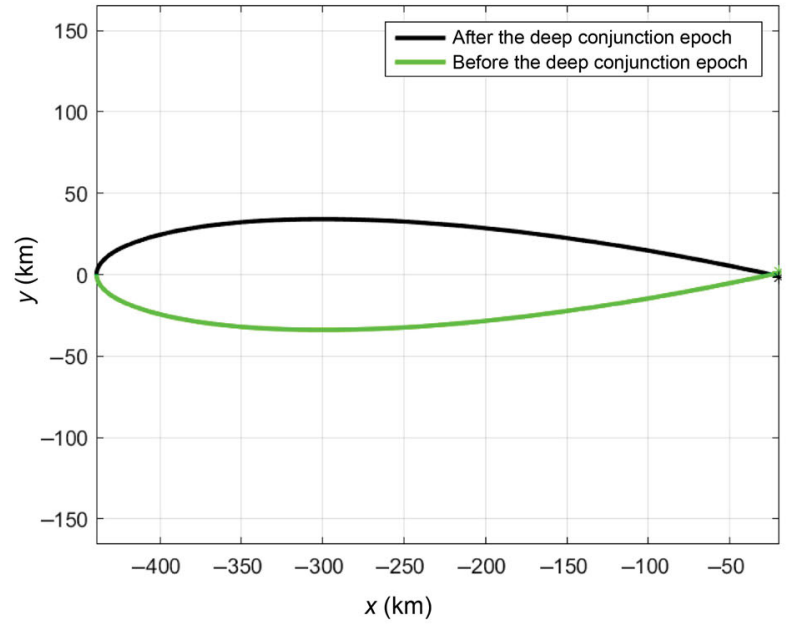

(a) in deep conjunction. A radio science experiment was carried out during the deep conjunction epoch (the green point in Fig. 1) for testing the Kaband capability for retrieving telemetry data to estimate the spacecraft's position and velocity. The spacecraft remained in deep conjunction for 21 days with no commands sent from Earth.

(3) Recovery phase: TCM2 (2018/12/22)-HRM $(2018 / 12 / 29)$. The recovery phase required a second TCM2 maneuver when the SEP angle was $4^{\circ}$. The HRM was performed when the SEP angles was $5^{\circ}$.

(4) Home position keeping: HPK (2018/12/29). At the HRM epoch, a $\Delta V$ for HPK maintenance was added with the scope of bringing the spacecraft to $20 \mathrm{~km}$ altitude on 2018/12/31.

\subsection{Maneuver operation}

The conjunction orbit took into account the solar radiation pressure perturbation and was designed to stay in the $+z_{\mathrm{HP}}$ region without any deterministic maneuvers between COI and HRM. The maximum distance from Ryugu was $109 \mathrm{~km}$ on 2018/12/11 (deep conjunction epoch, green point in Fig. 1). The COI and HRM $\Delta V \mathrm{~s}$ were $2 \mathrm{~cm} / \mathrm{s}$ in $x_{\mathrm{HP}}$ and $12 \mathrm{~cm} / \mathrm{s}$ in $y_{\mathrm{HP}}$. The maneuvers were calculated based on the results of the orbit determination (OD) team, who made use of radiometric data and ONC-W1's asteroid image tracking (AIT) data to estimate the state of the spacecraft. The AIT data was verified using the

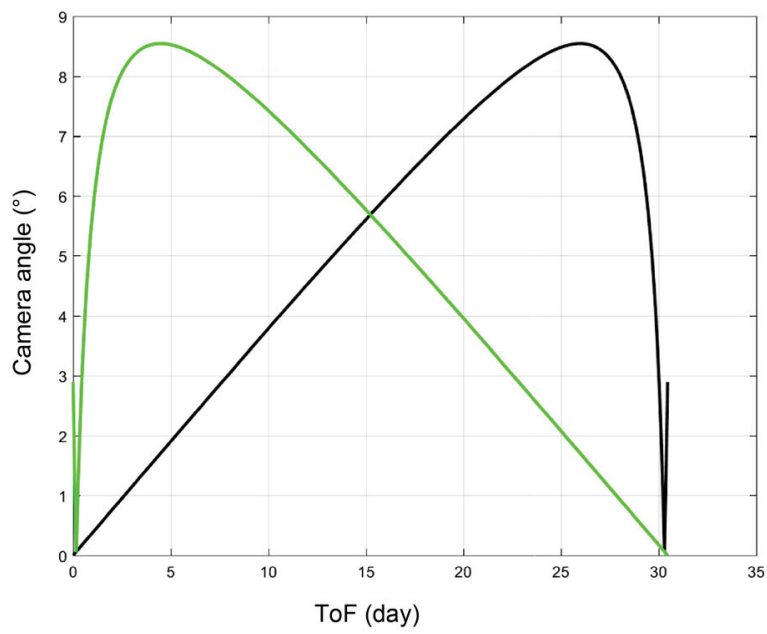

(b)

Fig. 2 The left panel shows the two arcs of the conjunction trajectory from deep conjunction to COI (green line) and from deep conjunction to HRM (black line). The right panel shows that Ryugu is always in the ONC-W1 camera FOV $\left(60^{\circ}\right)$ and in some cases in the FOV of the ONC-T camera $\left(6^{\circ}\right)$.

(10) UNIIVRSITY PRESA 
raw ONC-W1 images. Each maneuver was supported via 2 days of navigation campaign. The minimum $\Delta V$ threshold for the reaction control system (RCS) was $1 \mathrm{~mm} / \mathrm{s}$ and any maneuver below $1 \mathrm{~mm} / \mathrm{s}$ was cancelled. If the planned $\Delta V$ was above $10 \mathrm{~cm} / \mathrm{s}$ than the maneuver was divided into main and trim $\Delta V \mathrm{~s}$. The $\Delta V_{z_{\mathrm{HP}}}$ was measured by the 2-way Doppler, while the $\Delta V_{x, y_{\mathrm{HP}}}$ was measured by the accelerometers (ACMs). The trim, $\Delta V$, was also used as a minor correction of the main $\Delta V$ during the same pass (contingency case). The Hayabusa2 spacecraft was kept Earth-pointing during the conjunction phase, for radio-science purposes. The spacecraft made use of the star trackers to maintain its attitude. The attitude maneuvers were scheduled every three days to keep the high gain antenna (HGA) Earth-pointing. Every attitude slew was below $3^{\circ}$. The Earth moved at a rate of about $0.75\left(^{\circ}\right)$ /day and the half-band-width of the HGA was $1.2^{\circ}$. Note that it was verified that Ryugu was always visible from ONC-W1 $\left(60^{\circ} \mathrm{FOV}\right)$. During the entire sequence, the SEP angle was below $2^{\circ}$.

Figure 3 shows a schematic representation of the operation planing. Similar to Hayabuas2's approach phase [11], the operation planning for the solar conjunction phase can be divided into: Phase (1), onboard image-based optical measurements (before 7:00 UTC in Fig. 3); Phase (2), radio-optical hybrid navigation (7:00-13:00 UTC in Fig. 3); Phase (3), guidance (13:00-16:00 UTC in Fig. 3); and Phase (4), spacecraft operation (from 16:00 UTC in Fig. 3).

The operation planning started two days before the maneuver planning, known as the observation campaign, by downloading the telemetry from the spacecraft; Phase (1) in Fig. 3. The range-rate (RARR) and the

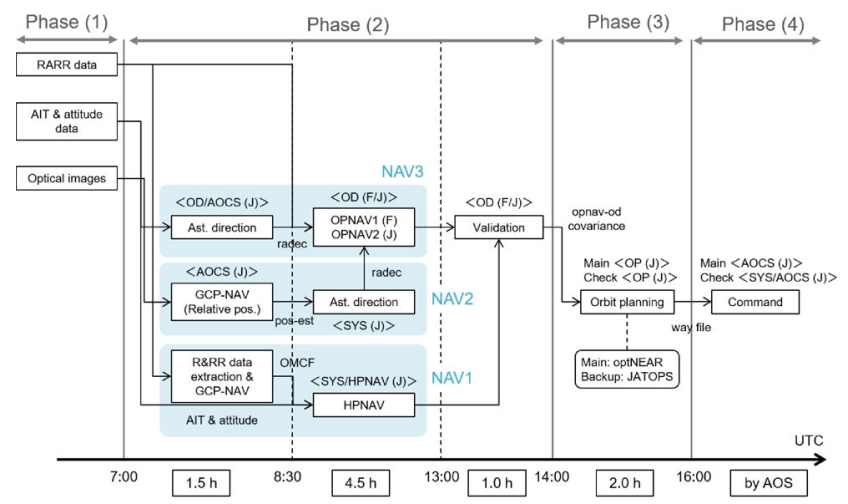

Fig. 3 Mission operation plan for each of the four scheduled conjunction maneuvers. onboard camera-based asteroid direction determination (asteroid image tracking-AIT) were combined $[6,7,11]$.

To guarantee a precise relative navigation, three techniques were run in parallel. These are shown in Fig. 3, Phase (2): (a) NAV1, called HPNAV (home position navigation), a hybrid navigation technique that combines radiometric (RARR) and optical navigation (ONC-T camera) techniques. It is a method for finding the position and speed of the spacecraft, using the direction to the image center and attitude data [11]. (b) NAV2, called GCP-NAV (ground control point navigation), a technique of finding the position and speed of the spacecraft by observing features on the asteroid surface [11]. (c) NAV3, a full asteroidspacecraft simultaneous orbit determination technique (Fujitsu team and JAXA team) [11].

Once the results of the navigation were validated, the $\Delta V$ planning phase started (Phase (3) in Fig. 3). The optNEAR tool was used as the main baseline for the $\Delta V$ planning, which is the subject of this article. The JATOPS tool, which was used during the approach phase, now became the backup solution for the solar conjunction phase [11]. The results of the guidance are given in a .way file format to be used as inputs to the spacecraft operation (Phase (4) in Fig. 3).

Finally, the spacecraft operation phase consisted of transforming the way file, $\Delta V$, from the HP reference frame to the spacecraft's asteroid fixed frame $[6,7]$ and sending the command to the spacecraft. The operation planning and the results of the mission operations are presented in this article. The mission operation process described here was followed for each of the four scheduled maneuvers at COI, TCM1, TCM2, and HRM epochs described in Table 1.

\section{$2.2 \quad$ Attitude maintenance}

The Hayabusa2 team considered three options for the attitude maintenance of the spacecraft during deep conjunction:

1) Safe mode (spinning). This method is safe as the spacecraft is passively stabilized. Only range and range rate are possible in this mode. However, its major drawback is it requires fuel and time to de-spin the spacecraft and progress to three-axis stabilisation.

2) Hayabusa2 acting as a solar sail. This mode makes use of 1 reaction wheel control $[12,13]$. The spacecraft is passively stabilized using the SRP

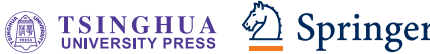


torque. The passive stabilisation makes this method very safe to use. This method was inspired by JAXA's IKAROS mission [14] and the Hayabusa2 spacecraft tested this method during cruise mode [12]. However, the major disadvantage of this method is that the HGA can't be used when the spacecraft is in "solar sail" mode as the spacecraft would need to be maintained as Sun-pointing, not Earth-pointing [13].

3) Hayabusa2 is kept Earth-pointing during deep conjunction. The spacecraft makes use of the star trackers to maintain its attitude. Attitude maneuvers are required during deep conjunction, which makes this method less safe than both options 1) and 2). However, the HGA can be used without any difficulties.

Since the Hayabusa2 team selected option 3) for radioscience purposes (testing of the Ka-band capability in deep conjunction) [15], attitude maneuvers were scheduled every three days to maintain the HGA as Earth-pointing.

\section{$3 n$-body propagator in J2000EQ coordinates centered at Ryugu (J2000EQ-Ry): optNEAR tool}

The optNEAR tool is a trajectory optimizer that make use of an $n$-body propagator written in J2000 equatorial coordinates, with the reference frame centered on Ryugu (J2000EQ-Ry). The optNEAR's propagator (known as goNEAR $[6,16])$ was written in python language and makes use of NASA's SPICE Toolkit package to import the ephemeris of Ryugu, all the planets, the Earth, Moon, and Sun. The effect of the SRP acceleration was also taken into account. In this case, the spacecraft was considered as Earth-pointing and the flat plate model was used for the SRP acceleration [17]. The $n$-body planetary equations are given by

$$
\left\{\begin{array}{c}
\dot{X} \\
\dot{Y} \\
\dot{Z} \\
\ddot{X} \\
\ddot{Y} \\
\ddot{Z}
\end{array}\right\}=\left[\begin{array}{c}
\dot{X} \\
\dot{Y} \\
\dot{Z} \\
-\frac{\mu_{\mathrm{a}}}{r^{3}} X+\left.\sum_{j=1}^{N P_{j}} a_{P}\right|_{x_{j}}+\left.a_{\mathrm{SRP}}\right|_{x} \\
-\frac{\mu_{\mathrm{a}}}{r^{3}} Y+\left.\sum_{j=1}^{N P_{j}} a_{P}\right|_{y_{j}}+\left.a_{\mathrm{SRP}}\right|_{y} \\
-\frac{\mu_{\mathrm{a}}}{r^{3}} Z+\left.\sum_{j=1}^{N P_{j}} a_{P}\right|_{z_{j}}+\left.a_{\mathrm{SRP}}\right|_{z}
\end{array}\right]=\left[\begin{array}{c}
F_{1} \\
F_{2} \\
F_{3} \\
F_{4} \\
F_{5} \\
F_{6}
\end{array}\right]
$$

or written more compactly:

$$
\dot{\boldsymbol{X}}=\boldsymbol{F}(\boldsymbol{X}, t)
$$

where $\mu_{a}$ is the gravity constant of Ryugu $\left(30 \mathrm{~m}^{3} / \mathrm{s}^{2}\right)$. The 3rd body acceleration is given by

$$
\boldsymbol{a}_{\mathrm{Pj}}=-\mu_{\mathrm{Pj}}\left(\frac{\boldsymbol{\Delta}}{\Delta^{3}}+\frac{\boldsymbol{d}}{d^{3}}\right)
$$

with $\boldsymbol{\Delta}=\boldsymbol{r}-\boldsymbol{d}$, where $\boldsymbol{r}$ is the spacecraft's position vector from Ryugu and $\boldsymbol{d}$ is the position vector of the perturbing body $(\mathrm{Pj})$ from Ryugu. Note that when the optNEAR tool calls the NASA's SPICE Toolkit, the ephemeris are downloaded from a reference frame centered on the solar system barycenter (SSB), and therefore the vector $\boldsymbol{d}$ is given by the position vector of the planet in SSB coordinates minus the position vector of Ryugu in SSB coordinates. For a non-diffusive Earthtracking flat surface, the SRP acceleration is

$\boldsymbol{a}_{\mathrm{SRP}}=-\frac{P_{0}}{c} \frac{A}{m}\left(\frac{A U}{r_{\mathrm{ls}}}\right)^{2} \cos \theta\left((1-\epsilon) \frac{\boldsymbol{r}_{\mathrm{ls}}}{r_{\mathrm{ls}}}+2 \epsilon \cos \theta \hat{\boldsymbol{n}}\right)$

where the Sun-line direction $\left(\boldsymbol{r}_{\mathrm{ls}}\right)$ is given by considering the distance of the spacecraft from Ryugu minus the distance of the Sun from Ryugu. The normal vector $(\hat{\boldsymbol{n}})$ to Hayabusa2's solar panels is kept Earth-pointing, thus

$$
\hat{\boldsymbol{n}}=\frac{\boldsymbol{r}_{\text {Earth }}}{r_{\text {Earth }}}
$$

and

$$
\cos \theta=\frac{\boldsymbol{r}_{\mathrm{ls}} \cdot \boldsymbol{r}_{\text {Earth }}}{r_{\mathrm{ls}} r_{\text {Earth }}}
$$

$\boldsymbol{r}_{\text {Earth }}$ is the Ryugu-Earth distance where the vector is pointing toward the Earth. In Eq. (4), $A$ is the spacecraft's reflective area, assumed as $13.276 \mathrm{~m}^{2}$ (i.e., the solar panels), the spacecraft's mass, $m$, is $580 \mathrm{~kg}$, $P_{0}$ is the solar flux of $1366 \mathrm{~W} / \mathrm{m}^{2}, c$ is the speed of light of $2.99792458 \times 10^{8} \mathrm{~m} / \mathrm{s}$, and $\epsilon$ is the reflectivity of the spacecraft, assumed to be 0.321. Note that $\epsilon=C_{\mathrm{r}}-1$, with $C_{\mathrm{r}}$ being the reflectivity coefficient of the spacecraft $(\epsilon=0$ complete absorption and $\epsilon=1$ complete specular reflection). A very simple way to demonstrate the relationship between $\epsilon$ and $C_{\mathrm{r}}$ is to consider that $\rho_{\mathrm{s}}+\rho_{\mathrm{a}}+\rho_{\mathrm{d}}=1$, with $\rho_{\mathrm{s}}$ being the specular reflectivity coefficient, $\rho_{\mathrm{a}}$ the absorption coefficient, and $\rho_{\mathrm{d}}$ the diffusive coefficient. If the diffusion term is neglected $\left(\rho_{\mathrm{d}}=0\right)$, it is possible to write that $\rho_{\mathrm{a}}=1-\rho_{\mathrm{s}}$ and also that $C_{\mathrm{r}}=1+\rho_{\mathrm{s}} \cdot \rho_{\mathrm{s}}$ and $\rho_{\mathrm{a}}$ were renamed here as $\epsilon$ and $C_{\mathrm{r}}$, respectively. The linearized equations of 
Eq. (1) are

$$
\dot{\boldsymbol{x}}(t)=\boldsymbol{A}(t) \boldsymbol{x}(t)
$$

where the matrix of the linearized equation can be derived as

$$
\boldsymbol{A}=\left[\begin{array}{cccccc}
0 & 0 & 0 & 1 & 0 & 0 \\
0 & 0 & 0 & 0 & 1 & 0 \\
0 & 0 & 0 & 0 & 0 & 1 \\
\frac{\partial F_{4}}{\partial x} & \frac{\partial F_{4}}{\partial y} & \frac{\partial F_{4}}{\partial z} & 0 & 0 & 0 \\
\frac{\partial F_{5}}{\partial x} & \frac{\partial F_{5}}{\partial y} & \frac{\partial F_{5}}{\partial z} & 0 & 0 & 0 \\
\frac{\partial F_{6}}{\partial x} & \frac{\partial F_{6}}{\partial y} & \frac{\partial F_{6}}{\partial z} & 0 & 0 & 0
\end{array}\right]
$$

The derivatives in Eq. (8) were computed analytically and their equations are given in Appendix A. To relate a state to a specific epoch, $t$, from an initial state, $t_{0}$, the state transition matrix is needed:

$$
\boldsymbol{\Phi}\left(t, t_{0}\right)=\frac{\partial \boldsymbol{X}(t)}{\partial \boldsymbol{X}\left(t_{0}\right)}
$$

that it is numerically computed as

$$
\dot{\boldsymbol{\Phi}}\left(t, t_{0}\right)=\boldsymbol{A}(t) \boldsymbol{\Phi}\left(t, t_{0}\right)
$$

with $\boldsymbol{\Phi}\left(t_{0}, t_{0}\right)=\boldsymbol{I}$. Therefore,

$$
\delta \boldsymbol{x}(t)=\boldsymbol{\Phi}\left(t, t_{0}\right) \delta \boldsymbol{x}\left(t_{0}\right)
$$

The computation of the STM can be done by deriving the analytic expression of the linearized equations matrix $\boldsymbol{A}$ and by solving Eq. (10) numerically, together with the equations of motion in Eq. (3). The derivatives in Appendix A were tested by comparing the analytical derivatives with the numerical derivatives.

\section{4 optNAER's single shooting method for the $\Delta V$ planning}

The shooting methods developed in the optNEAR tool were used for the $\Delta V$ planning during Hayabusa2's superior solar conjunction operation. The aim was to minimize the overall $\Delta V$ budget required to place the spacecraft in the ayu conjunction trajectory. The operation aimed to depart from the hovering location at HP and return to HP after the spacecraft left the Sun's shadow. optNEAR's shooting method aimed to minimize the $\Delta V$ maneuver such that following the integration of the non-linear dynamics in Eq. (1), the spacecraft returned to HP at the end of the solar conjunction. To achieve high accuracy in the final error position at HP, a constrained optimization was used such that:

$$
\min _{x, \alpha, \delta}|\Delta V|
$$

with

$$
\left\{\begin{array}{l}
V_{x}=V \cos \delta \cos \alpha \\
V_{y}=V \cos \delta \sin \alpha \\
V_{z}=V \sin \delta
\end{array}\right.
$$

where $V=V_{\max }(1+\sin x)$ and $\Delta V=\sqrt{V_{x}^{2}+V_{y}^{2}+V_{z}^{2}}$, subject to the following constraints:

$$
\begin{aligned}
& \left|x\left(t_{1}\right)-\bar{x}\right|-\text { toll }=0 \\
& \left|y\left(t_{1}\right)-\bar{y}\right|-\text { toll }=0 \\
& \left|z\left(t_{1}\right)-\bar{z}\right|-\text { toll }=0
\end{aligned}
$$

The toll is usually set to $0.1 \mathrm{~m}$. Note that the minimization of $\Delta V$ is reduced to finding three angles, $\alpha$ (in-plane angle), $\delta$ (out-of-plane angle), and $x$ (e.g., $V=V_{\max }$, with $x=0^{\circ}$ ). At $t_{1}$, after the ODE integration of Eq. (1), the final desired position of the spacecraft was to be equal to the nominal state $\overline{\boldsymbol{r}}_{\mathrm{HP}}=[0,0,20 \mathrm{~km}]$, in HP coordinates, at the end of the conjunction epoch $(2018 / 12 / 29$ in Table 1$)$. Note that the ODE integration was performed in the J2000EQRy reference frame, and therefore a transformation was required to move $\overline{\boldsymbol{r}}_{\mathrm{HP}}$ into J2000EQ-Ry coordinates $(\overline{\boldsymbol{r}})$, as shown in Soldini et al. $[6,7]$.

\subsection{Deterministic $\Delta V$ maneuver at COI: Refinement of the Hill trajectory in the $n$-body dynamics}

At the beginning of the conjunction phase (COI epoch in Table 1), the shooting method (optNEAR tool) described made use of the ayu conjunction trajectory designed in the Hill coordinates [6, 7]. The ayu trajectory designed in Refs. [6,7] was therefore the first guess for the two-boundary value problem in the full ephemeris model (goNEAR tool $[6,16]$ ) where the initial (COI) and final (HRM) positions were fixed. In Fig. 4, the black line is the un-optimized trajectory (goNEAR propagator), while the red trajectory is optimized with the optNEAR tool. The magenta point is the location of the HP at HRM (2018/12/29 in Table 1). Tables 2 and 3 show the $\Delta V$ designed at COI and HRM in the HP and J2000EQ-Ry coordinates, respectively. Each table shows the epoch of the maneuver in UTC and the $\Delta V$ is 


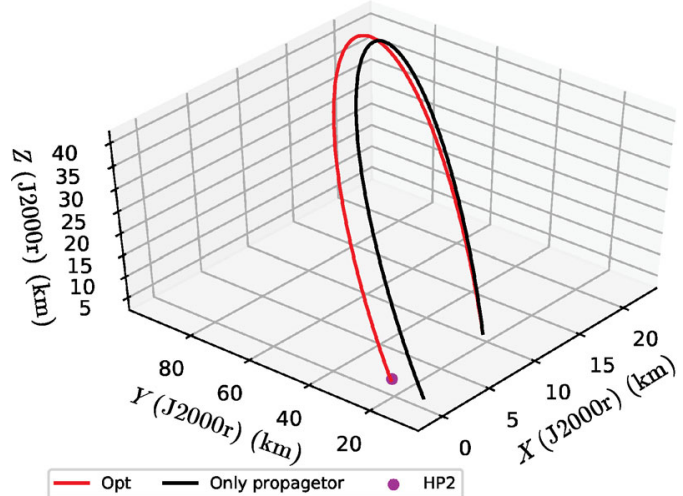

Fig. 4 Conjunction trajectory optimized (red) with the $n$-body optimizer (optNEAR tool) and the propagated trajectory in black (goNEAR tool) as seen from Ryugu, Eq. (1).

given for each axis direction in HP coordinates (Table 2) and in J2000 coordinates (Table 3). Note that due to the mounting direction of the thrusters a $\Delta V$ margin (Table 2) was added to include thrust losses in the $y_{\mathrm{HP}}$ direction $[6,7]$. The settings used in the optNEAR tool for the COI maneuver planning were $V_{\max }=0.0004$ $\mathrm{km} / \mathrm{s}$. The lower and the upper boundaries of the angles were set as: $x\left(-90^{\circ}, 0^{\circ}\right), \alpha\left(0^{\circ}, 30^{\circ}\right)$, and $\delta\left(0^{\circ}, 90^{\circ}\right)$.

\subsection{Trajectory correction maneuvers: TCM1 and TCM2}

After COI, every initial state guess at the TCM epoch $t_{0}$ (time of maneuver) can be found analytically through the state transition matrix:

$$
\left\{\begin{array}{l}
\delta \boldsymbol{r}_{1} \\
\delta \boldsymbol{v}_{1}
\end{array}\right\}=\left[\begin{array}{ll}
\boldsymbol{\Phi}_{11} & \boldsymbol{\Phi}_{12} \\
\boldsymbol{\Phi}_{21} & \boldsymbol{\Phi}_{22}
\end{array}\right]_{t_{1}, t_{0}}\left\{\begin{array}{c}
\delta \boldsymbol{r}_{0} \\
\delta \boldsymbol{v}_{0}
\end{array}\right\}
$$

with the objective of bringing the final position state to zero, as shown in Fig. $5(\mathrm{~b})\left(\delta \boldsymbol{r}_{0}=0\right)$. The first equation

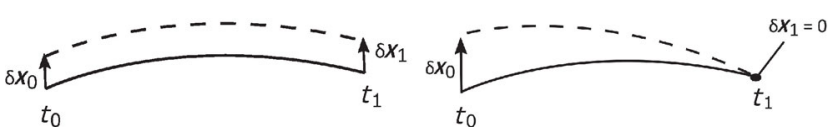

$\begin{array}{ll}\text { (a) State transition matrix } & \text { (b) Desired final position at } t_{1}\end{array}$

Fig. 5 Shooting method: reference trajectory (solid line) and perturbed trajectory (dashed line).

in the system of Eq. (15) is

$$
\boldsymbol{\Phi}_{11}\left(t_{1}, t_{0}\right) \delta \boldsymbol{r}_{0}+\boldsymbol{\Phi}_{12}\left(t_{1}, t_{0}\right) \delta \boldsymbol{v}_{0}=\mathbf{0}
$$

so that

$$
\delta \boldsymbol{v}_{0}=-\boldsymbol{\Phi}_{12}^{-1}\left(t_{1}, t_{0}\right) \boldsymbol{\Phi}_{11}\left(t_{1}, t_{0}\right) \delta \boldsymbol{r}_{0}
$$

with $\boldsymbol{\Phi}_{11}\left(t_{1}, t_{0}\right)$ as

$$
\boldsymbol{\Phi}_{11}\left(t_{1}, t_{0}\right)=\left[\begin{array}{lll}
\Phi_{11} & \Phi_{12} & \Phi_{13} \\
\Phi_{21} & \Phi_{22} & \Phi_{23} \\
\Phi_{31} & \Phi_{32} & \Phi_{33}
\end{array}\right]_{\left(t_{1}, t_{0}\right)}
$$

and $\boldsymbol{\Phi}_{12}\left(t_{1}, t_{0}\right)$ as

$$
\boldsymbol{\Phi}_{12}\left(t_{1}, t_{0}\right)=\left[\begin{array}{lll}
\Phi_{14} & \Phi_{15} & \Phi_{16} \\
\Phi_{24} & \Phi_{25} & \Phi_{26} \\
\Phi_{34} & \Phi_{35} & \Phi_{36}
\end{array}\right]_{\left(t_{1}, t_{0}\right)}
$$

The initial state is therefore

$$
\left\{\begin{array}{l}
\boldsymbol{x}_{0}=\delta \boldsymbol{x}_{0}+\overline{\boldsymbol{x}}_{0} \\
\boldsymbol{v}_{0}=\delta \boldsymbol{v}_{0}+\overline{\boldsymbol{v}}_{0}
\end{array}\right.
$$

and is used as the initial guess for the shooting method

\begin{tabular}{|c|c|c|c|c|c|}
\hline & $\begin{array}{l}\text { Epoch } \\
(\mathrm{ET})\end{array}$ & $\begin{array}{c}\text { UTC } \\
\text { (date) }\end{array}$ & $\begin{array}{c}\Delta V_{x}(\mathrm{HP}) \\
(\mathrm{m} / \mathrm{s})\end{array}$ & $\begin{array}{c}\frac{\Delta V_{y}(\mathrm{HP})}{\cos \left(75^{\circ}\right)} \\
(\mathrm{m} / \mathrm{s})\end{array}$ & $\begin{array}{c}\Delta V_{z}(\mathrm{HP}) \\
(\mathrm{m} / \mathrm{s})\end{array}$ \\
\hline $\mathrm{COI}$ & $596,203,269.18$ & 2018/11/23 Т00:00 & $1.891 \mathrm{E}-02$ & $-6.0322 \mathrm{E}-03$ & $1.175 \mathrm{E}-01$ \\
\hline HRM & $599,313,669.18$ & 2018/12/29 Т00:00 & $-1.8058 \mathrm{E}-02$ & $1.5744 \mathrm{E}-02$ & $-1.1549 \mathrm{E}-01$ \\
\hline Total & & & $3.6977 \mathrm{E}-02$ & $2.177 \mathrm{E}-02$ & $2.3299 \mathrm{E}-01$ \\
\hline
\end{tabular}
in optNEAR. The settings used for the optNEAR tool at TCMs are

\begin{tabular}{|c|c|c|c|c|c|}
\hline & $\begin{array}{l}\text { Epoch } \\
(\mathrm{ET})\end{array}$ & $\begin{array}{l}\text { UTC } \\
\text { (date) }\end{array}$ & $\begin{array}{c}\Delta V_{x}(\mathrm{~J} 2000) \\
(\mathrm{m} / \mathrm{s})\end{array}$ & $\begin{array}{c}\Delta V_{y}(\mathrm{~J} 2000) \\
(\mathrm{m} / \mathrm{s})\end{array}$ & $\begin{array}{c}\Delta V_{z}(\mathrm{~J} 2000) \\
(\mathrm{m} / \mathrm{s})\end{array}$ \\
\hline COI & $596,203,269.18$ & 2018/11/23 Т00:00 & $3.1793 \mathrm{E}-02$ & $1.05487 \mathrm{E}-01$ & $4.50463 \mathrm{E}-02$ \\
\hline $\mathrm{HRM}$ & $599,313,669.18$ & 2018/12/29 Т00:00 & $1.4375 \mathrm{E}-02$ & $1.07179 \mathrm{E}-01$ & $4.4575 \mathrm{E}-02$ \\
\hline Total & & & $4.6168 \mathrm{E}-02$ & $2.12666 \mathrm{E}-01$ & $8.9621 \mathrm{E}-02$ \\
\hline
\end{tabular}

a) TCM1 maneuver settings:

$V_{\max }$ was set to $0.002 \mathrm{~km} / \mathrm{s}$. The lower and the upper

Table $2 \Delta V$ in HP reference frame for the Earth-pointing spacecraft $\left(\mu_{a}=32 \mathrm{~m}^{3} / \mathrm{s}^{2}\right.$ and SEP $\left.=5^{\circ}\right)$

Table $3 \Delta V$ in $\mathrm{J} 2000$ reference frame for the Earth-pointing spacecraft $\left(\mu_{a}=32 \mathrm{~m}^{3} / \mathrm{s}^{2}\right.$ and $\left.\mathrm{SEP}=5^{\circ}\right)$ 
boundaries of the angles were set as: $x\left(-90^{\circ}, 0^{\circ}\right)$, $\alpha\left(0^{\circ}, 30^{\circ}\right)$, and $\delta\left(0^{\circ}, 90^{\circ}\right)$;

b) TCM2 maneuver settings:

$V_{\max }$ was set to $0.01 \mathrm{~km} / \mathrm{s}$. The lower and the upper boundaries of the angles were set as: $x\left(-90^{\circ}, 0^{\circ}\right)$, $\alpha\left(-90^{\circ}, 0^{\circ}\right)$, and $\delta\left(-180^{\circ}, 0^{\circ}\right)$.

\subsection{Brake velocity maneuver at HRM and HPK maneuver}

Planning for the $\Delta V$ maneuver at HRM included both a brake velocity maneuver at HP on 2018/12/29 and a HPK maneuver for BOX-A operation maintenance, until $2018 / 12 / 31$. The first $\Delta V$ aimed to simply stop the spacecraft at the HP arrival point (HRM). The HPK maneuver required designing in the Hill coordinates, as for the ayu conjunction trajectory. The same shooting method described in Soldini et al. [6, 7] was used but with the following initial guess: $H_{0}=25 \mathrm{~km}$ (maximum altitude), $\alpha_{0}=188^{\circ}$ (in-plane angle in the $x-y_{\text {Hill }}$ coordinates), and $v_{z_{0}}=0 \mathrm{~km} / \mathrm{s}$ (out of plane velocity). The lower and upper boundaries of the optimum parameters were as follows: $20 \mathrm{~km}<H<$ $30 \mathrm{~km}, 180^{\circ}<\alpha<270^{\circ}$, and $-0.00001 \mathrm{~km} / \mathrm{s}<v_{z}<$ $0.00001 \mathrm{~km} / \mathrm{s}$. Once the HPK maintenance arc was designed in the Hill reference frame, the solution was refined in the optNEAR tool; $V_{\max }$ was set to 0.0002 $\mathrm{km} / \mathrm{s}$ and the lower and upper boundaries of the angles were set as follows: $x\left(-90^{\circ}, 0^{\circ}\right), \alpha\left(0^{\circ}, 30^{\circ}\right)$, and $\delta\left(90^{\circ}\right.$, $180^{\circ}$ ). Figure 6 shows the nominal HPK arc trajectory designed with the optNEAR tool, if the HRM point is in its nominal location of $20 \mathrm{~km}$ altitude.

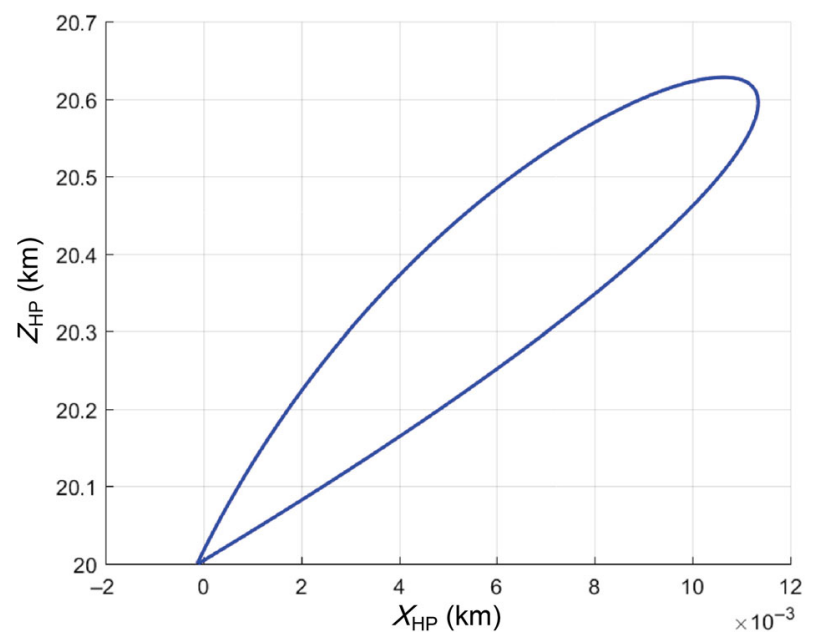

Fig. 6 Example of the HPK trajectory arc in the HP reference frame, from $2018 / 12 / 29$ to $2018 / 12 / 31$.

\section{JAXA's JATOPS tool: The backup solution of the optNEAR tool}

As part of the $\Delta V$ planning at COI, TCM1, TCM2, and HRM, the optNEAR tool was used as the main solution for planning the $\Delta V$ command, which was to be executed on board the spacecraft. However, it was further verified that JAXA's trajectory optimisation tool, JATOPS [11], could retrieve the same solution as the optNEAR tool, once the nominal states at the COI, TCM1, TCM2, and HRM epochs were computed by optNEAR. The difference between optNEAR and JATOPS is in the ability to design the ayu trajectory in a single shooting. OptNEAR implements a semianalytical method that uses the weak stability boundary theory to design the first guess trajectory in one pass (the ayu trajectory). Once optNEAR has successfully provided the first guess (the ayu solution), JATOPS can then be used as a validation tool for the $\Delta V$ s computed with optNEAR. For further details on the JATOPS tool, refer to Tsuda et al. [11].

Figure 7 shows that the JATOPS tool could be used for high altitude operations as it finds the same solutions as the optNEAR tool [11]. Therefore, our $\Delta V$ planning strategy was to use the optNEAR tool as a baseline for the computation of the $\Delta V$ commands and to rely on the JATOPS tool as a back-up solution. The JATOPS tool was selected as a back-up solution for the optNEAR tool and the following procedure to retrieve the nominal trajectory with JATOPS was confirmed [11]:

(1) The ayu trajectory was divided into three trajectory legs: COI-TCM1 (Leg1), TCM1-TCM2 (Leg2), and

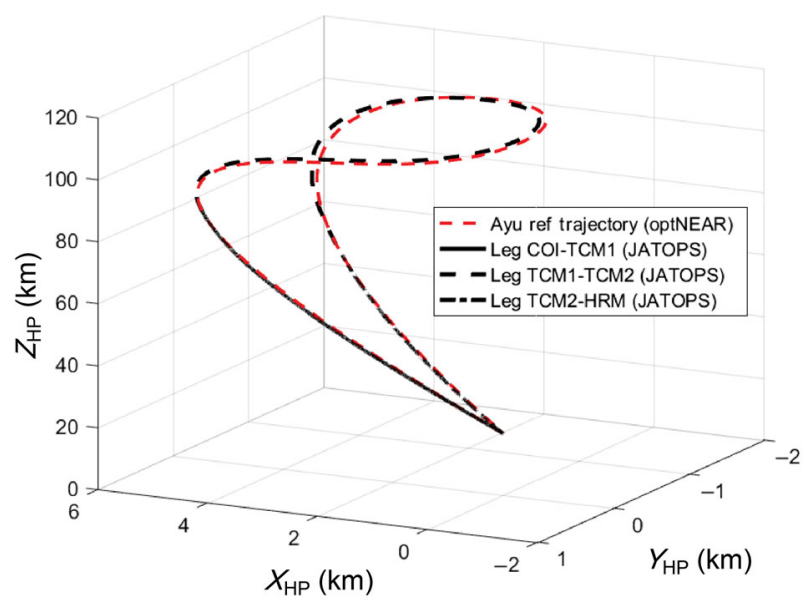

Fig. 7 The conjunction trajectory as seen from the HP frame: a comparison between the solutions obtained between the optNEAR (red line) and the JATOPS (black line) tools. 
TCM2-HRM (Leg3).

(2) Each leg derived a two-impulse trajectory, using states derived by the optNEAR tool from the output .way file as the boundary conditions.

(3) It was confirmed that the JATOPS tool was a good back-up in extreme cases when a $\Delta V$ was required to safely return back to HP.

(4) The JATOPS tool does not have the capability to instantaneously derive the entire nominal ayu conjunction orbit from COI to HRM, as with the optNEAR tool.

\section{Post mission operation}

In this section, each of the four maneuvers performed during the solar conjunction phase are presented. The solar conjunction operation was planned as described in Section 2 and in Fig. 3. For each operation planning (Fig. 3), first the results of the OD teams were analyzed. Once the most reliable estimate of the spacecraft's position and velocity was selected by the flight dynamic (FD) and OD teams, planning of the $\Delta V$ resulted in the delivery of the .way file from the FD team to the attitude orbit control system (AOCS) team. The $\Delta V$ was thus given as a sequence of commands to the spacecraft. After the $\Delta V$ was executed on board the spacecraft, its velocity (2-way Doppler) and acceleration (ACMs) were measured to estimate the actual $\Delta V$ performed. The actual and the planned $\Delta V \mathrm{~s}$ were thus compared to evaluate the performance of the operation. In case of large discrepancies between $\Delta V \mathrm{~s}$, it was possible to correct the maneuver within the same communication pass. At the end of the operation, the measured $\Delta V$ was used for the trajectory design of the next trajectory leg.

\subsection{Conjunction orbit insertion (COI) maneuver: 2018/11/23}

On 2018/11/23, the Hayabusa2 spacecraft performed the conjunction orbit insertion maneuver. The navigation and operation planning and their results are presented here. The $\Delta V$ planning started on 2018/11/22 after two days of data measurements. The initial downlink of the telemetry data started on $2018 / 11 / 21$.

(a) Once the downlink of the AIT and Doppler data was concluded, the navigation teams performed an estimate of the spacecraft's position and velocity. The navigation team's estimates at COI are shown in Table 4. Those estimates were compared with the nominal case, as shown in the first row of Table 4 . From those estimates, the corresponding $\Delta V$ s were computed, as shown in Table 5.

To select the best estimate of the spacecraft's state from the JAXA and Fujitsu solutions, the $\Delta V \mathrm{~s}$ in Table 5 were cross evaluated with the different solutions in Table 4. The solutions were thus propagated with goNEAR.

From this analysis, the solution from Fujitsu was selected for the $\Delta V$ planning as it was shown to be the most conservative solution in the presence of uncertainties in the navigation.

(b) After having selected the estimate from the OD's Fujitsu team, the FD team prepared the $\Delta V$ planning at COI. Figure 8 shows the way file prepared on 2018/11/22 for the following day's operation. The .way file was the input for the AOCS team, where the $\Delta V$ was computed in a body-fixed frame. Figure 8 shows the estimated states at each epoch and the corresponding $\Delta V \mathrm{~s}$.

(c) The error between the planned and the actual $\Delta V$ was of $98.78 \%$ in $\Delta V_{X_{B}}, 93.53 \%$ in $\Delta V_{Y_{B}}$, and $98.53 \%$ in $\Delta V_{Z_{B}}$, as shown in the report of Fig. 9. Figure 9 shows the measured and planned $\Delta V \mathrm{~s}$ for the main $(\mathrm{M})$ and trim $(\mathrm{T})$ maneuvers for each axis direction. The accuracy in the maneuvers in term of absolute and relative errors are also provided. Due to uncertainties in the $\Delta V$ at COI, it was concluded that at least one TCM was required on

Table 4 Nominal state vector at COI and estimated states by Fujitsu (OPNAV1/NAV3 in Fig. 3), JAXA (OPNAV2/NAV3 in Fig. 3), and HPNAV (NAV1 in Fig. 3)

\begin{tabular}{ccccccc}
\hline $\begin{array}{c}\text { State } \\
\text { estimate }\end{array}$ & $\begin{array}{c}x_{\mathrm{HP}} \\
(\mathrm{km})\end{array}$ & $\begin{array}{c}y_{\mathrm{HP}} \\
(\mathrm{km})\end{array}$ & $\begin{array}{c}z_{\mathrm{HP}} \\
(\mathrm{km})\end{array}$ & $\begin{array}{c}v_{x_{\mathrm{HP}}} \\
(\mathrm{mm} / \mathrm{s})\end{array}$ & $\begin{array}{c}v_{y_{\mathrm{HP}}} \\
(\mathrm{mm} / \mathrm{s})\end{array}$ & $\begin{array}{c}v_{z_{\mathrm{HP}}} \\
(\mathrm{mm} / \mathrm{s})\end{array}$ \\
\hline Nominal & 0 & 0 & 20 & 0 & 0 & 0 \\
OD's Fujitsu & -0.0115 & -0.1428 & 20.0692 & -1.7101 & 1.2674 & -17.9384 \\
OD's JAXA & -0.0458 & -0.1197 & 19.9301 & -2.1938 & 1.4375 & -17.9382 \\
HPNAV & 0.0425 & -0.1217 & 19.7622 & -1.7098 & 1.7039 & -18.0709 \\
\hline
\end{tabular}




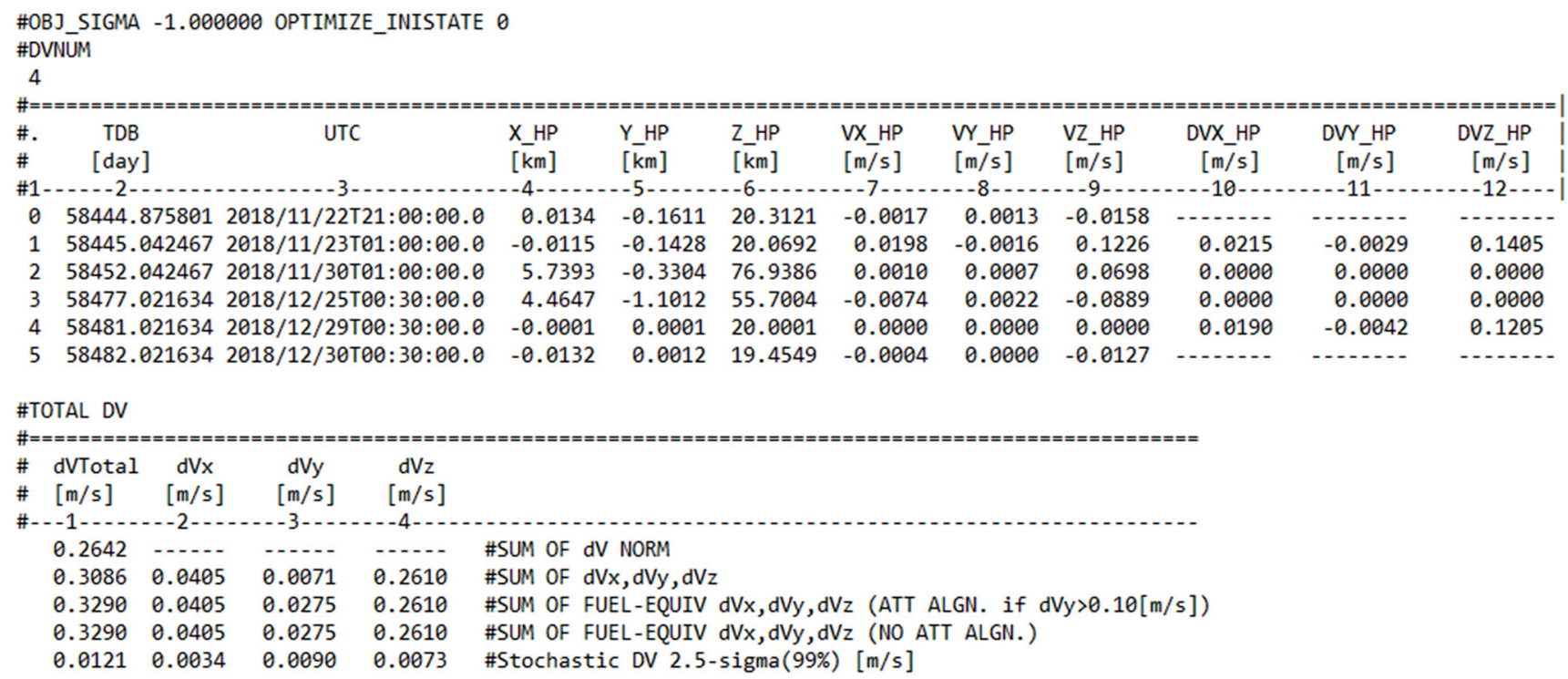

Fig. 8 Planned $\Delta V$ at COI in .way file format.

Table 5 Nominal $\Delta V$, OD's Fujitsu $\Delta V$ (OPNAV1/NAV3 in Fig. 3), and OD's JAXA $\Delta V$ (OPNAV2/NAV3 in Fig. 3)

\begin{tabular}{cccc}
\hline $\begin{array}{c}\text { State } \\
\text { estimate }\end{array}$ & $\begin{array}{c}\Delta V_{X_{\mathrm{HP}}} \\
(\mathrm{cm} / \mathrm{s})\end{array}$ & $\begin{array}{c}\Delta V_{Y_{\mathrm{HP}}} \\
(\mathrm{cm} / \mathrm{s})\end{array}$ & $\begin{array}{c}\Delta V_{Z_{\mathrm{HP}}} \\
(\mathrm{cm} / \mathrm{s})\end{array}$ \\
\hline Nominal & 1.9844 & -0.1637 & 12.2624 \\
OD's Fujitsu & 2.1548 & -0.2906 & 14.0497 \\
OD's JAXA & 2.2060 & -0.3080 & 14.0625 \\
\hline
\end{tabular}

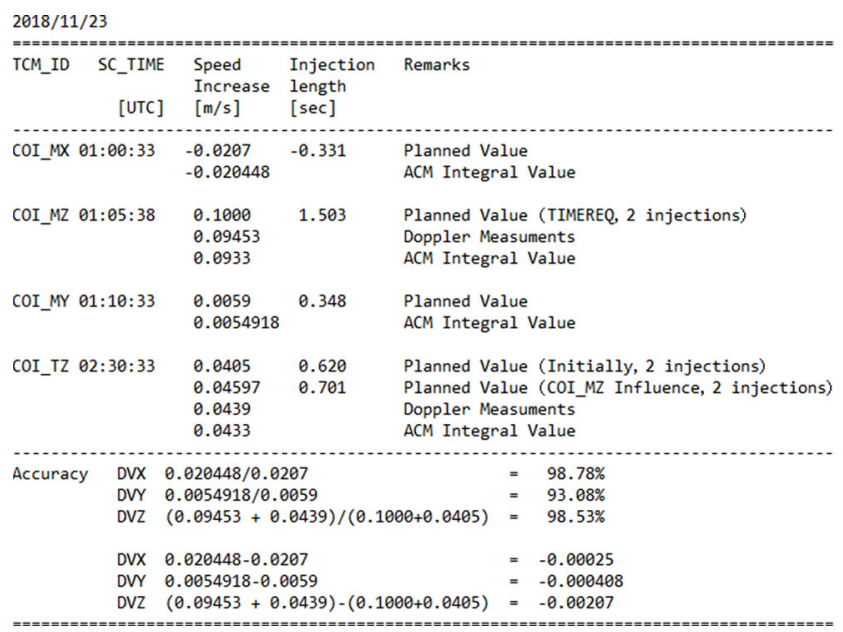

Fig. 9 Results of the COI operation on 2018/11/23: planned and measured $\Delta V$ report.

2018/11/30 before deep conjunction. Errors in the $\Delta V$ lower than $4 \mathrm{~mm} / \mathrm{s}$ were considered acceptable, as concluded in Soldini et al. [6,7], where it was shown that those errors resulted in a negligibly small position error of the spacecraft. For this reason, no contingency $\Delta V$ was required at COI. It was also expected that the major $\Delta V$ at TCM1 would have been in the $Z$ component.

Figure 10 shows the planned trajectory for the selected solution (OD's Fujitsu is shown in black). The red trajectory leg is the predicted trajectory from COI to TCM1, when the actual $\Delta V$ from the result report in Fig. 9 was used. The green trajectory is the new solution from TCM1 to HRM, designed with the optNEAR tool.

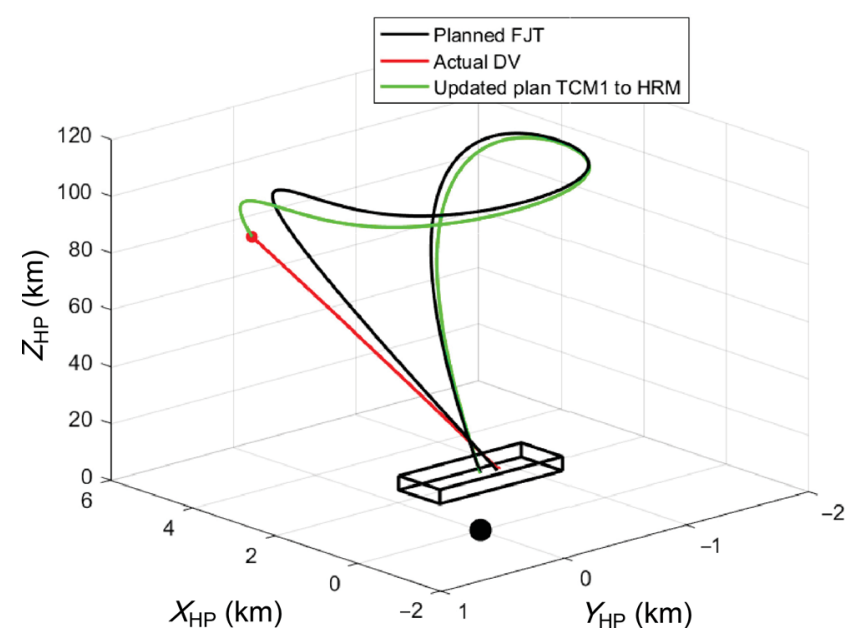

Fig. 10 Planned trajectory OD Fujitsu solution (black), actual trajectory from COI to TCM1 after COI operation (red), and new planned trajectory between TCM1 to HRM (green). The black dot represents the Ryugu coordinates. HP reference frame. 


\subsection{Trajectory correction maneuver 1 (TCM1): 2018/11/30}

The first trajectory correction maneuver (TCM1) was performed on 2018/11/30 before the spacecraft entered the deep solar conjunction phase, with the SEP angle equal to $3^{\circ}$. The TCM1 allowed corrections in the trajectory after the actual operation at COI. The $\Delta V$ at TCM1 was planned on 2018/11/29 after the initial downlink of the telemetry data on $2018 / 11 / 28$.

(a) The estimates of the spacecraft's position and velocity on 2018/11/29 are shown in Table 6 .

As with COI, the solutions were crosschecked with the nominal case designed after the COI maneuver (the green trajectory in Fig. 10). The $\Delta V \mathrm{~s}$ correspondence with the estimated solutions are shown in Table 7 . As with the COI maneuver planning, the $\Delta V \mathrm{~s}$ in Table 7 were cross evaluated with the different solutions in Table 6 and the solutions were propagated with goNEAR. From this analysis, the solution from JAXA was selected for the $\Delta V$ planning at TCM1.

(b) Figure 11 shows the .way file prepared on the 2018/11/29 for the TCM1 operation (2018/11/30).
The OD's JAXA team's .way file was the input for the AOCS team and the $\Delta V$ was computed in a body-fixed frame.

(c) In the case of TCM1, the $\Delta V_{X}$ and $\Delta V_{Y}$ were cancelled, resulting in an error after 24 days of 1 $\mathrm{km}$ in $\mathrm{X}_{\mathrm{HP}}$ at TCM2, as shown in Fig. 13. An increase in the noise of the Doppler signal already at TCM1 was registered, as shown in Fig. 12. The noise is evident by looking at the fluctuation in the vertical axis of Fig. 12, which represents the double error between the measured and planned speed of the spacecraft. This fluctuation is in the order of the size of the maneuvers $(\mathrm{mm} / \mathrm{s})$ and is usually a straight line, when the Sun corona is out of the way of the communication link. The report in Fig. 14 shows that the error in the $\Delta V_{Z_{B}}$ was $99.21 \%$, resulting in a perfectly executed operation. Between TCM1 and TCM2, only pre-scheduled attitude maintenance maneuvers were performed (every 1-2 days). It was decided to avoid desaturation of the reaction wheels in deep conjunction to prevent errors to the planned trajectory between TCM1 and TCM2.

Table 6 Nominal state vector at TCM1 and estimated states by Fujitsu (OPNAV1/NAV3 in Fig. 3), JAXA (OPNAV2/NAV3 in Fig. 3), and HPNAV (NAV1 in Fig. 3)

\begin{tabular}{|c|c|c|c|c|c|c|}
\hline $\begin{array}{c}\text { State } \\
\text { estimate }\end{array}$ & $\begin{array}{l}x_{\mathrm{HP}} \\
(\mathrm{km})\end{array}$ & $\begin{array}{c}y_{\mathrm{HP}} \\
(\mathrm{km})\end{array}$ & $\begin{array}{c}z_{\mathrm{HP}} \\
(\mathrm{km})\end{array}$ & $\begin{array}{c}v_{x_{\mathrm{HP}}} \\
(\mathrm{mm} / \mathrm{s})\end{array}$ & $\begin{array}{c}v_{y_{\mathrm{HP}}} \\
(\mathrm{mm} / \mathrm{s})\end{array}$ & $\begin{array}{c}v_{z_{\mathrm{HP}}} \\
(\mathrm{mm} / \mathrm{s})\end{array}$ \\
\hline Nominal & 5.6589 & -0.0395 & 75.1693 & 1.0321 & 1.1463 & 67.1665 \\
\hline OD's Fujitsu & 5.6086 & -0.3308 & 75.0280 & 0.5944 & 0.6297 & 67.0149 \\
\hline OD's JAXA & 5.6395 & -0.3308 & 74.7831 & 0.8847 & 0.6304 & 67.0185 \\
\hline HPNAV & 5.8616 & -0.4094 & 74.8879 & 0.9457 & 0.6333 & 68.0690 \\
\hline
\end{tabular}

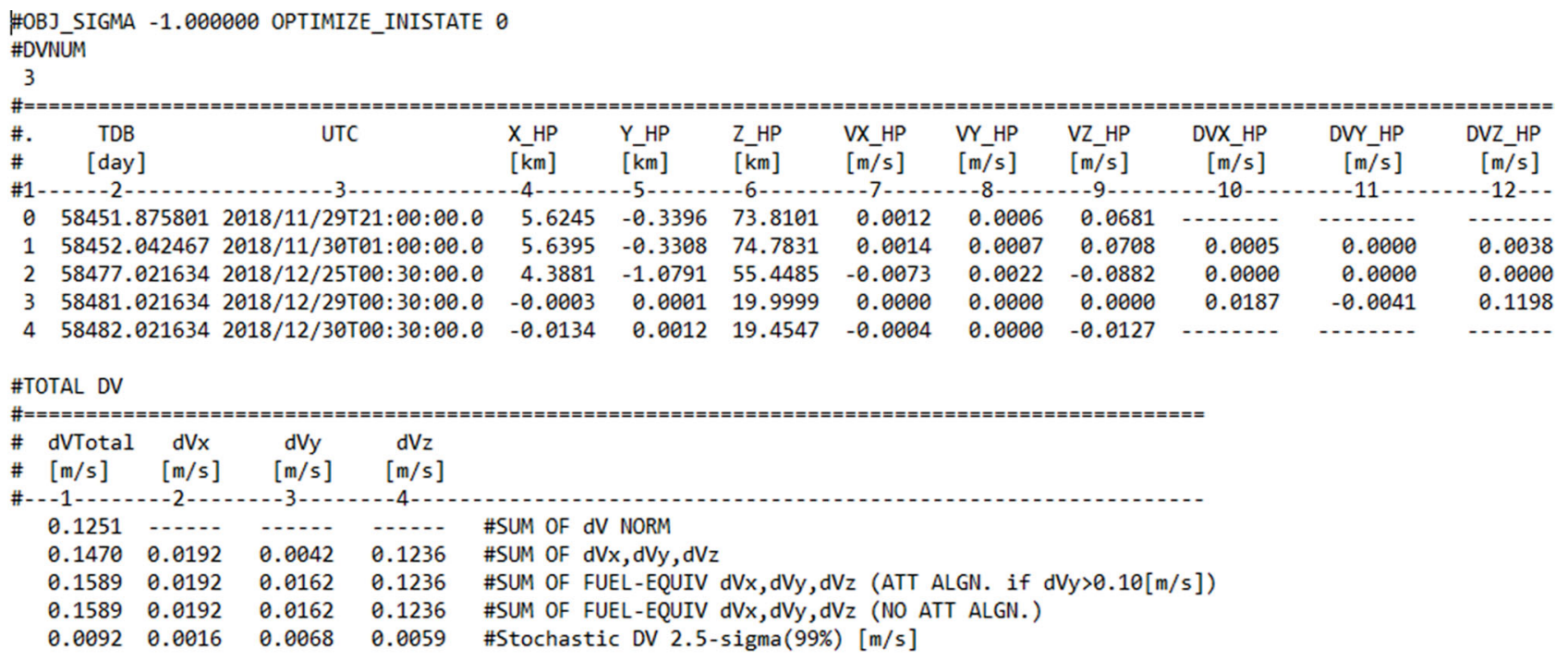

Fig. 11 Planned $\Delta V$ at TCM1 in .way file format. 
Table 7 Nominal $\Delta V$, OD's Fujitsu $\Delta V$ (OPNAV1/NAV3 in Fig. 3), and OD's JAXA $\Delta V$ (OPNAV2/NAV3 in Fig. 3)

\begin{tabular}{cccc}
\hline $\begin{array}{c}\text { State } \\
\text { estimate }\end{array}$ & $\begin{array}{c}\Delta V_{X_{\mathrm{HP}}} \\
(\mathrm{cm} / \mathrm{s})\end{array}$ & $\begin{array}{c}\Delta V_{Y_{\mathrm{HP}}} \\
(\mathrm{cm} / \mathrm{s})\end{array}$ & $\begin{array}{c}\Delta V_{Z_{\mathrm{HP}}} \\
(\mathrm{cm} / \mathrm{s})\end{array}$ \\
\hline Nominal & 0.0230 & -0.0573 & 0.3460 \\
OD's Fujitsu & 0.0752 & 0.0039 & 0.3674 \\
OD's JAXA & 0.0492 & 0.0042 & 0.3793 \\
\hline
\end{tabular}

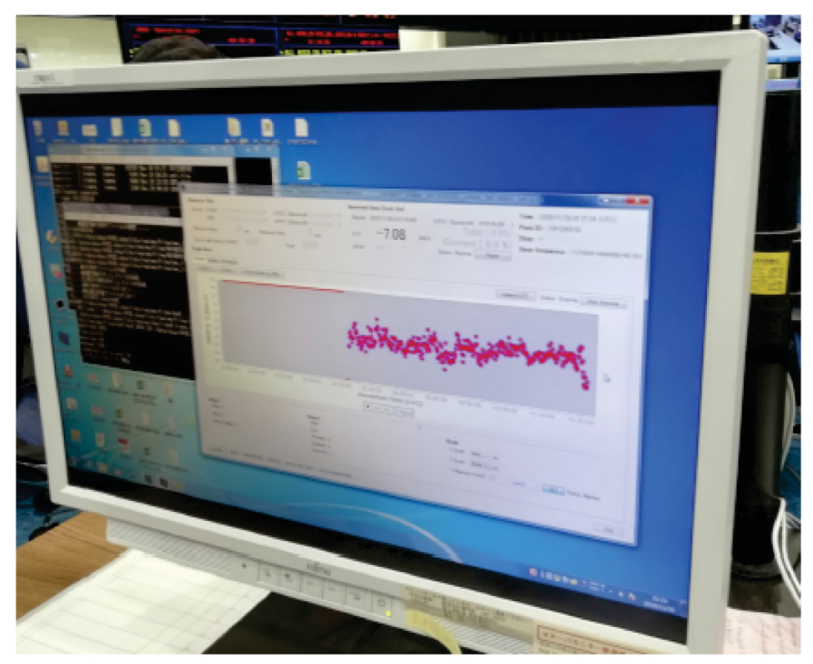

Fig. 12 Doppler signal on 2018/11/30. Horizontal axis: reception time in UTC. Vertical axis: the double difference between measured and planned value of the spacecraft's speed.

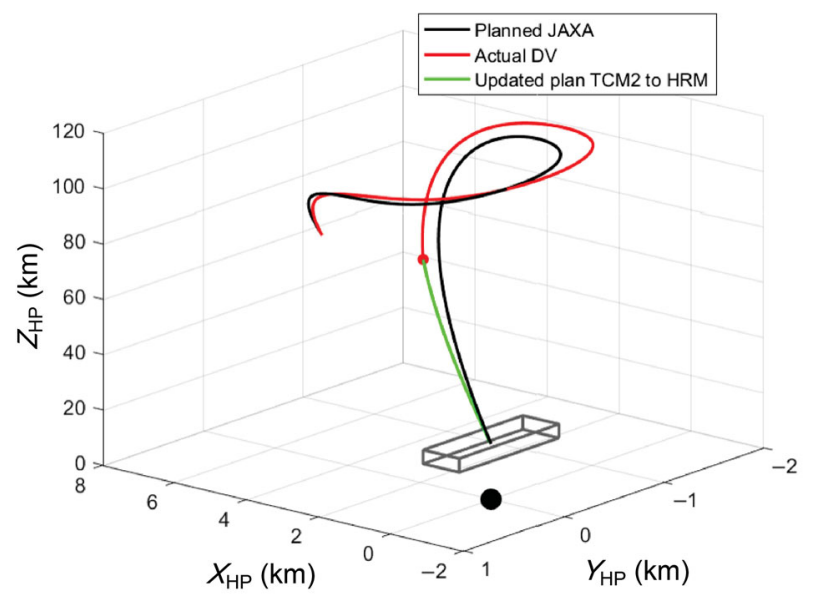

Fig. 13 Planned trajectory OD JAXA solution (black), actual trajectory from TCM1 to TCM2 after the TCM1 operation (red), and new planned trajectory between TCM2 to HRM (green). The black dot represents the Ryugu coordinates. HP reference frame.

\subsection{Deep conjunction epoch: 2018/12/11}

During the deep conjunction epoch on 2018/12/11, beacon operations were performed to test the Ka-band capability and estimate the state vector of Hayabusa2 [15]; refer to the green dot in Fig. 1 for the deep conjunction epoch. The propagated trajectory after

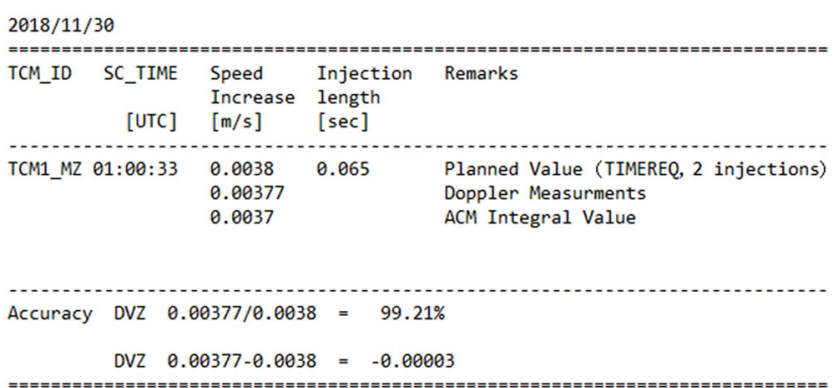

Fig. 14 Results of the TCM1 operation on 2018/11/30: planned and measured $\Delta V$ report.

estimating the state vector on $2018 / 12 / 11$ is shown in Fig. 15, in gray. Compared to the propagated trajectory after executing the actual $\Delta V$ at TCM1, a displacement in position of $2 \mathrm{~km}$ in the $X_{\mathrm{HP}}$ direction at TCM2 was noticed. It was verified that the $0.5 \mathrm{~mm} / \mathrm{s}$ correction not given at TCM1 in the $X$-axis was one of the causes of this expected position displacement at TCM2. Moreover, the trajectory between TCM1 and TCM2, designed with the optNEAR tool, assumed that the spacecraft was always Earth-pointing. However, attitude maneuvers were scheduled every 3 days, which resulted in an error in the pointing accuracy of $1^{\circ}$; consequently, the effect on the SRP acceleration was weaker. A smaller effect on the SRP acceleration caused a drift in the $X$-axis direction away from the asteroid, as shown in Fig. 16. It was verified that propagating the planned trajectory with a reflective coefficient, $C_{\mathrm{r}}$, of $5 \%$ less than the nominal value would have compensated for the error in the $X_{\mathrm{HP}}$ position, shown by the purple line in Fig. 16. Furthermore, it is possible that nonlinearities affected our solution after 21 days. Therefore, a TCM2 maneuver was needed immediately after deep conjunction on 2018/12/25.

The error in the attitude is also thought to be the reason why the asteroid was not in the FOV of the ONCT camera on 2018/12/15-17, as shown in Fig. 17. Figure 17(b) shows that the state of the spacecraft could not be estimated with the ONC-T camera as Ryugu was out of the camera's FOV, as determined from the images taken. Figure 18 shows the angle between Ryugu and the spacecraft each day after $2018 / 12 / 11$; it can be seen that when the error of $1^{\circ}$ in the attitude was included in the analysis, Ryugu was not in the FOV of the ONC$\mathrm{T}$ on either day, as shown in Fig. 18(b). Due to the geometry of the ayu trajectory, when the attitude error was not taken into account it is possible to notice that 


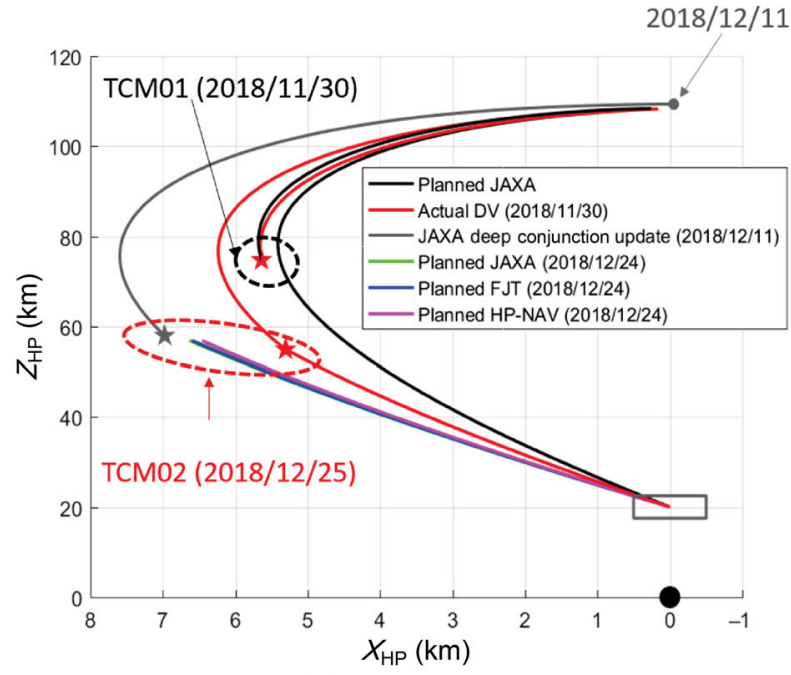

(a) $X-Z$ HP view

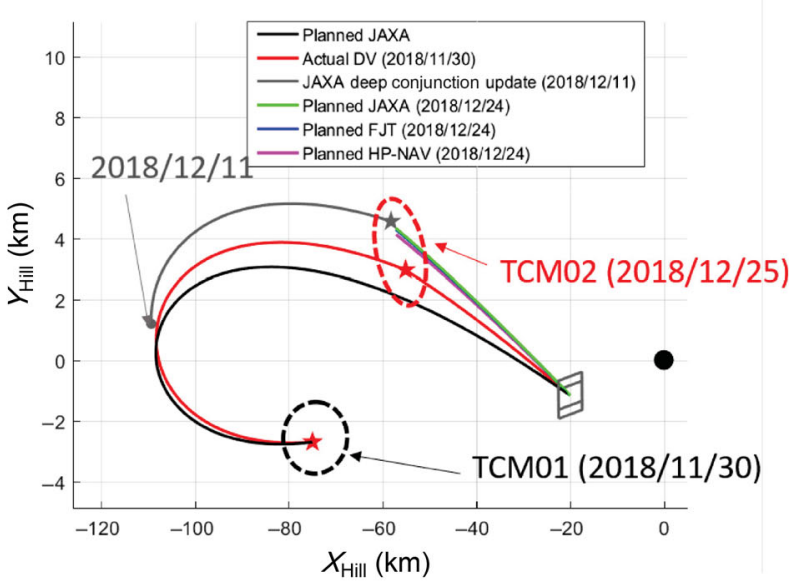

(b) $X-Y$ HP view

Fig. 15 Estimated trajectory in deep conjunction after beacon operation.

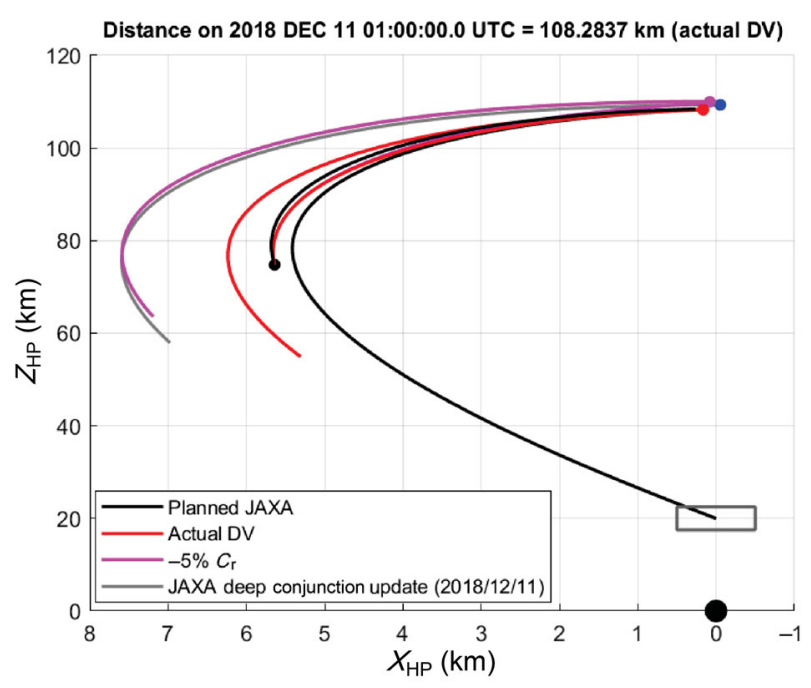

Fig. 16 Estimating the equivalent error in $C_{\mathrm{r}}$.
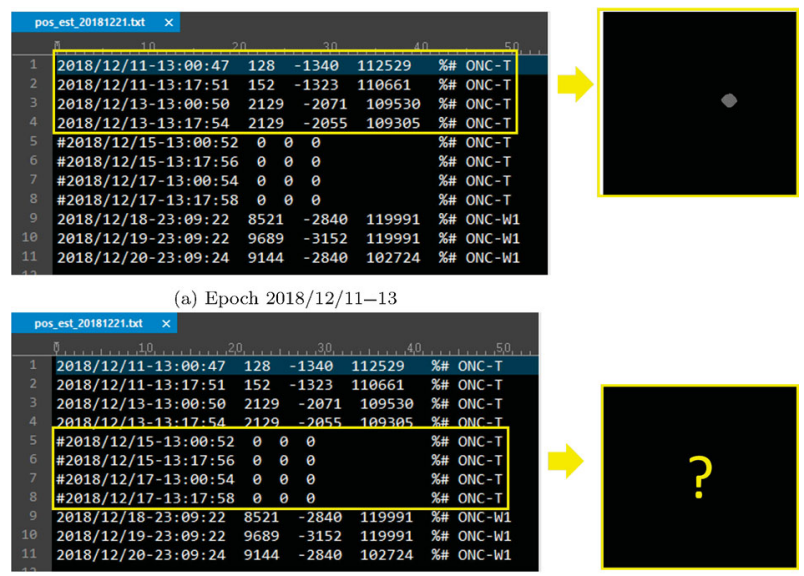

(b) Epoch 2018/12/15-17

Fig. 17 Post estimation 2018/12/21: ONC-T images.
Ryugu was not in the ONC-T's FOV on 2018/15/17, as shown in Fig. 18(a).

The observation campaign was started on 2018/12/21 to verify that the $Z_{\mathrm{HP}}$ altitude was within the expected values. From the Doppler signal in Fig. 19, it was verified that the spacecraft's velocity had changed sign (Fig. 19(b)), and that the spacecraft was returning to lower altitudes (Fig. 19(a)). The altitude of the spacecraft was approximately $82 \mathrm{~km}$ as planned in Fig. 19(a), and was decreasing towards the HP position altitude.

\subsection{Trajectory correction maneuver 2 (TCM2): 2018/12/25}

After 21 days of blackout in the communication link, the first telemetry data from the spacecraft was downloaded on 2018/12/22. The second TCM2 maneuver was scheduled for the $2018 / 12 / 25$ after the planning on 2018/12/24.

(a) The estimates of the spacecraft's position and velocity on 2018/12/24 are shown in Table 8 . The solutions for the COI and TCM1 operations planning were crosschecked with the nominal case, designed after the TCM1 maneuver (the green trajectory in Fig. 13) was executed. The $\Delta V \mathrm{~s}$ corresponding to the estimate solutions are shown in Table 9. The $\Delta V \mathrm{~s}$ in Table 9 were cross evaluated with the different solutions in Table 8 and the solutions were propagated with goNEAR. From this analysis, the solution from Fujitsu was selected for the $\Delta V$ planning at TCM2. 


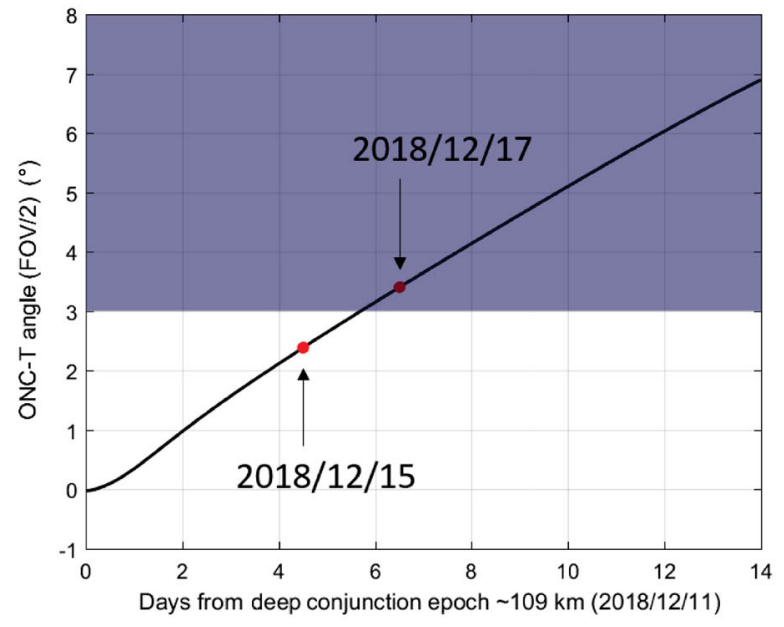

(a) No error in attitude

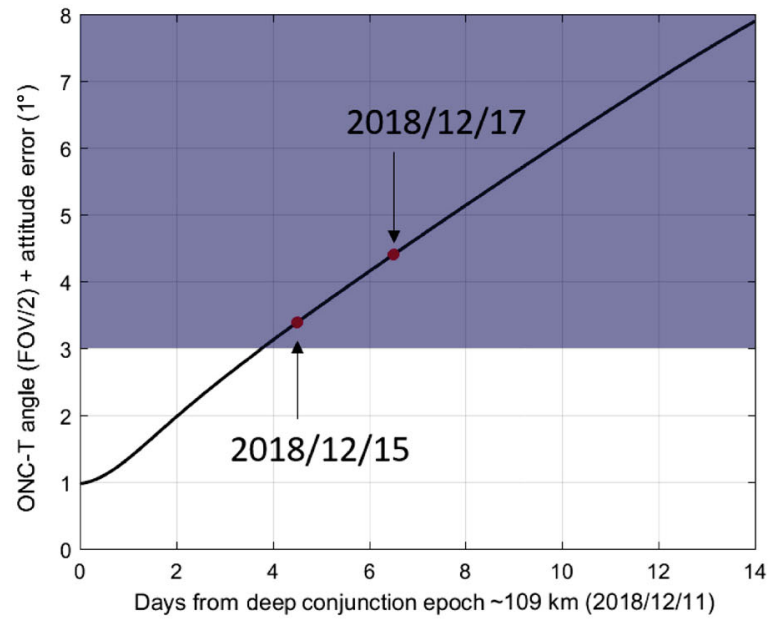

(b) Attitude error

Fig. 18 ONC-T angle FOV as function of the days from deep conjunction: the blue area shows during which epochs Ryugu is not in the FOV of ONC-T.

Table 8 Nominal state vector at TCM2 and estimated states by Fujitsu (OPNAV1/NAV3 in Fig. 3), JAXA (OPNAV2/NAV3 in Fig. 3), and HPNAV (NAV1 in Fig. 3)

\begin{tabular}{ccccccc}
\hline $\begin{array}{c}\text { State } \\
\text { estimate }\end{array}$ & $\begin{array}{c}x_{\mathrm{HP}} \\
(\mathrm{km})\end{array}$ & $\begin{array}{c}y_{\mathrm{HP}} \\
(\mathrm{km})\end{array}$ & $\begin{array}{c}z_{\mathrm{HP}} \\
(\mathrm{km})\end{array}$ & $\begin{array}{c}v_{x_{\mathrm{HP}}} \\
(\mathrm{mm} / \mathrm{s})\end{array}$ & $\begin{array}{c}v_{y_{\mathrm{HP}}} \\
(\mathrm{mm} / \mathrm{s})\end{array}$ & $\begin{array}{c}v_{z_{\mathrm{HP}}} \\
(\mathrm{mm} / \mathrm{s})\end{array}$ \\
\hline Nominal & 5.3185 & -1.2411 & 55.0592 & -0.6931 & 0.2238 & -8.8516 \\
OD's Fujitsu & 6.6334 & -1.8447 & 56.8919 & -0.6281 & 0.1917 & -8.6760 \\
OD's JAXA & 6.5968 & -1.8250 & 56.8739 & -0.6252 & 0.1940 & -8.6823 \\
HPNAV & 6.4577 & -1.7198 & 56.8581 & -0.8764 & 0.2514 & -8.7487 \\
\hline
\end{tabular}

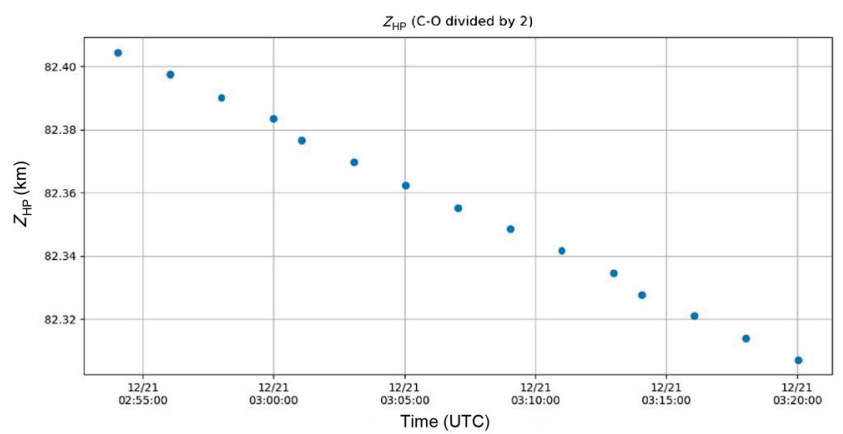

(a) Position

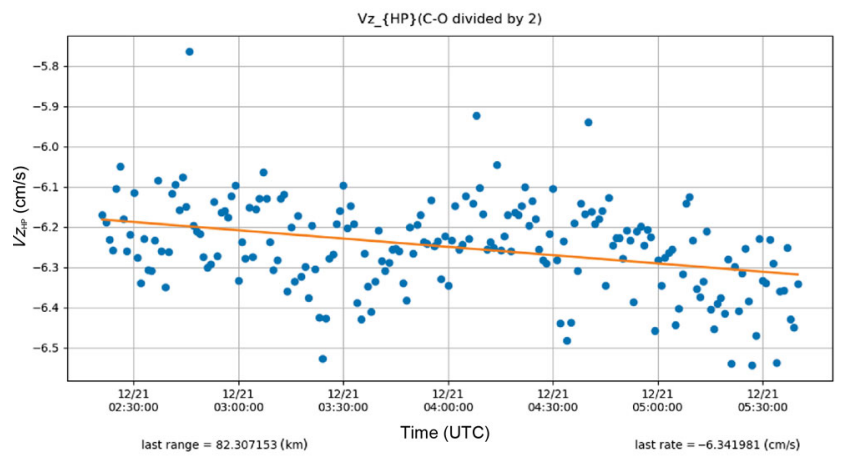

(b) Velocity

Fig. 19 Doppler measurements on 2018/12/21.
Table 9 Nominal $\Delta V$, OD's Fujitsu $\Delta V$ (OPNAV1/NAV3 in Fig. 3), and OD's JAXA $\Delta V$ (OPNAV2/NAV3 in Fig. 3)

\begin{tabular}{cccc}
\hline State & $\Delta V_{X_{\mathrm{HP}}}$ & $\Delta V_{Y_{\mathrm{HP}}}$ & $\Delta V_{Z_{\mathrm{HP}}}$ \\
estimate & $(\mathrm{cm} / \mathrm{s})$ & $(\mathrm{cm} / \mathrm{s})$ & $(\mathrm{cm} / \mathrm{s})$ \\
\hline Nominal & -3.0005 & 0.5207 & 1.5554 \\
OD's Fujitsu & -7.0870 & 2.6334 & -5.8566 \\
OD's JAXA & -7.0163 & 2.5521 & -5.7345 \\
\hline
\end{tabular}

(b) Figure 20 shows the .way file prepared on 2018/12/24 for the TCM2 operation (2018/12/25). The OD's Fujitsu team .way file was the input for the AOCS team and the $\Delta V$ was computed in a body-fixed frame.

(c) As a consequence of cancelling the $\Delta V_{X}$ and $\Delta V_{X}$ at TCM1, the TCM2 in each $X, Y$, and $Z$ component was performed, as shown in Fig. 20. As expected, the larger $\Delta V$ was given in the $X$ direction, as at TCM1 (Fig. 11). The $\Delta V_{X_{B}}$ of $0.5 \mathrm{~mm} / \mathrm{s}$ was not performed, resulting in an expected $1 \mathrm{~km}$ displacement in $X$ at TCM2. The error between the planned and the actual $\Delta V$ at TCM2 was $105.89 \%$ in $\Delta V_{X_{B}}, 100.21 \%$ in $\Delta V_{Y_{B}}$, and $93.48 \%$ in 


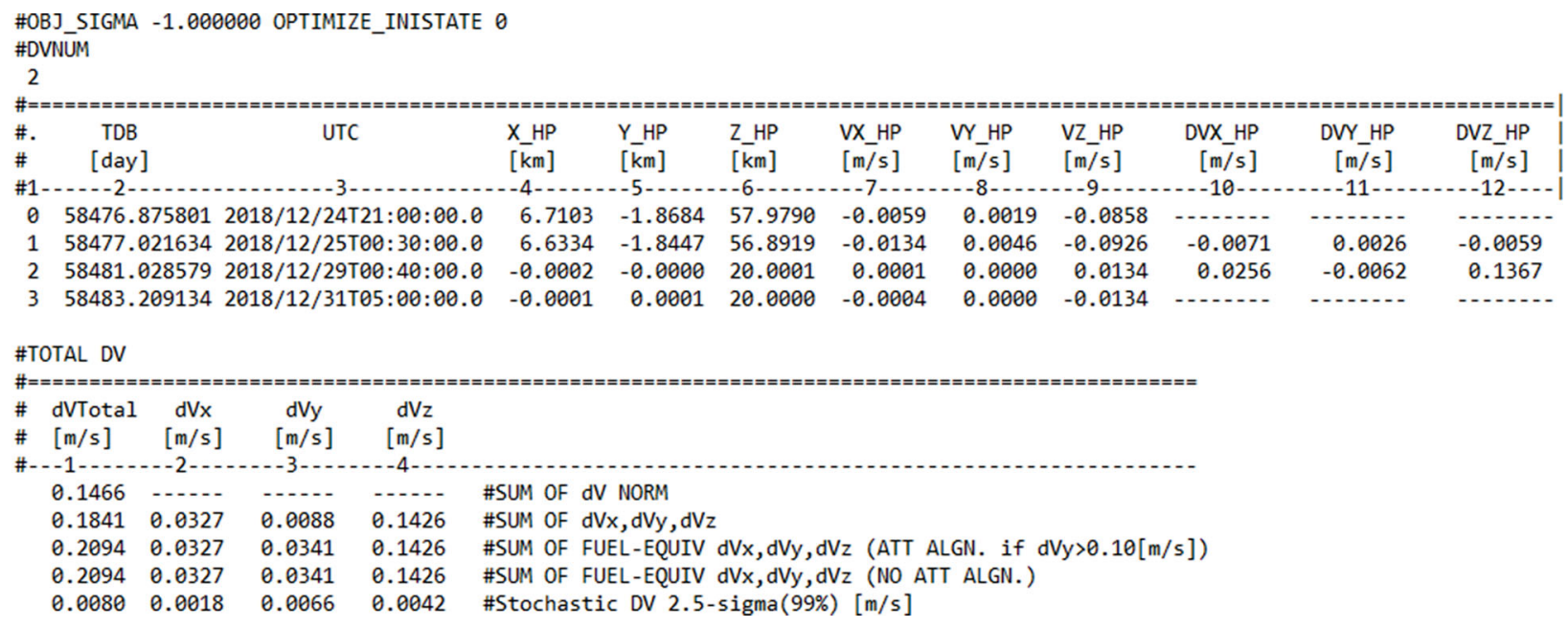

Fig. 20 Planned $\Delta V$ at TCM2 in .way file format.

$\Delta V_{Z_{B}}$, as shown in the report of Fig. 21. Figure 22 shows the $X-Z$ and $Y-Z$ components of the ayu trajectory in the HP frame at TCM2, displayed in the control room.

\begin{tabular}{|c|c|c|c|c|}
\hline TCM_ID & $\begin{array}{r}\text { SC_TIME } \\
\text { [UTC] }\end{array}$ & $\begin{array}{l}\text { Speed } \\
\text { Increase } \\
{[\mathrm{m} / \mathrm{s}]}\end{array}$ & $\begin{array}{l}\text { Injectior } \\
\text { length } \\
\text { [sec] }\end{array}$ & Remarks \\
\hline \multicolumn{2}{|c|}{ TCM2_MX $00: 35: 33$} & $\begin{array}{l}-0.007206 \\
-0.007631\end{array}$ & -0.126 & $\begin{array}{l}\text { Planned Value } \\
\text { ACM Integral Value }\end{array}$ \\
\hline TCM2_MZ & $00: 40: 38$ & $\begin{array}{l}-0.005892 \\
-0.005508 \\
-0.00702\end{array}$ & -0.113 & $\begin{array}{l}\text { Planned Value (TIMEREQ, } 2 \text { injections) } \\
\text { Doppler Measuments } \\
\text { ACM Integral Value }\end{array}$ \\
\hline TCM2_MY & $00: 45: 33$ & $\begin{array}{l}0.002310 \\
0.002315\end{array}$ & 0.147 & $\begin{array}{l}\text { Planned Value } \\
\text { ACM Integral Value }\end{array}$ \\
\hline \multirow[t]{4}{*}{ Accuracy } & $\begin{array}{l}\text { DVX } \\
\text { DVY } \\
\text { DVZ }\end{array}$ & $\begin{array}{l}0.007631 / \\
0.002315 / \\
0.005508 /\end{array}$ & $\begin{array}{l}0.007206 \\
0.002310 \\
0.005892\end{array}$ & $\begin{array}{l}=105.89 \% \\
=100.21 \% \\
=93.48 \%\end{array}$ \\
\hline & $\mathrm{DVX}$ & $0.007631-$ & 0.007206 & $=0.000425$ \\
\hline & DVY & $0.002315-$ & $0.002310=$ & $=0.000499$ \\
\hline & DVZ & $0.005508-$ & 0.005892 & $=-0.00038$ \\
\hline
\end{tabular}

Fig. 21 Results of the TCM2 operation on 2018/12/25: planned and measured $\Delta V$ report.

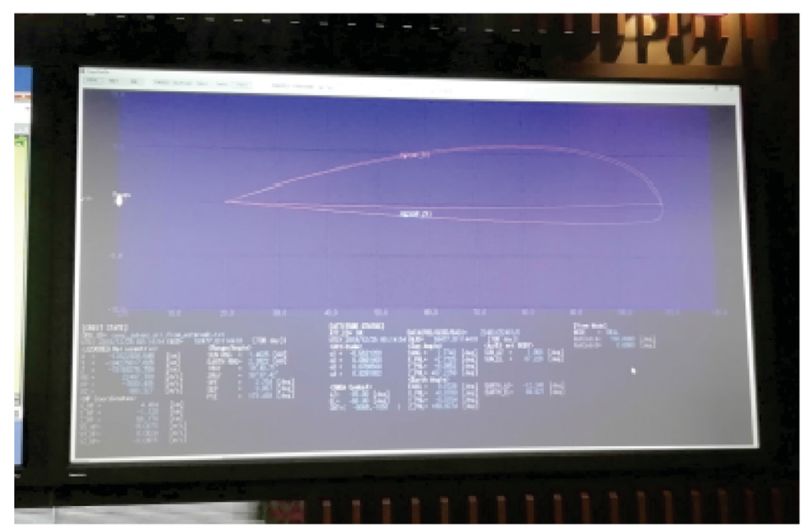

Fig. 22 Planned ayu trajectory displayed on the screen of the ISAS's control room on 2018/12/25.
Figure 23 shows the results after the operation by comparing the planned trajectory with the actual predicted trajectory, in black and red, respectively.

\subsection{Home recovery maneuver and home position keeping: 2018/12/29}

On 2019/12/29, the last conjunction operation was performed. As explained in Section 4.3, two $\Delta V \mathrm{~s}$ were combined into one maneuver; a HPK maneuver (BOX-A operation keeping until 2018/12/31) together with the HRM $\Delta V$.

(a) Table 10 shows the estimates of the spacecraft's position and velocity on 2018/12/30. As for the previous operations, the solutions were crosschecked with the nominal case, designed after the TCM2 maneuver (Fig. 23) was executed. The $\Delta V \mathrm{~s}$

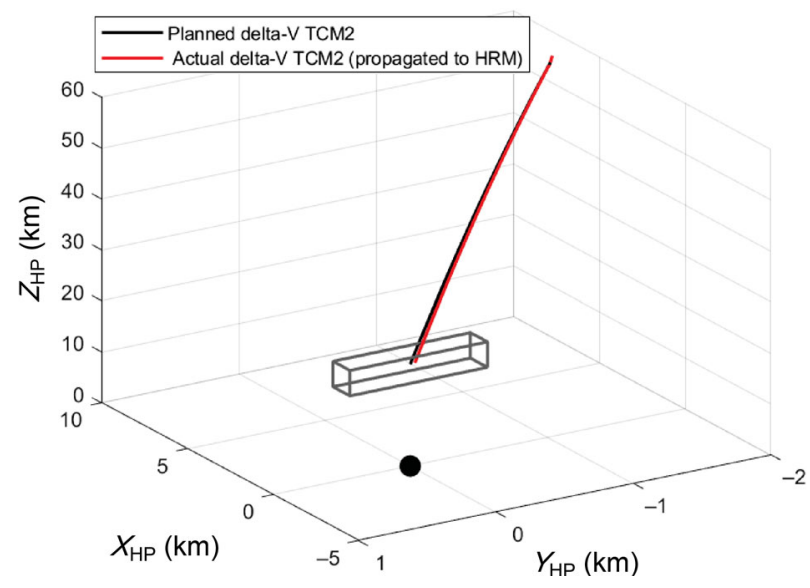

Fig. 23 Planned trajectory OD Fujitsu solution (black), actual trajectory after TCM2 operation (red), and BOX-A operation (gray). The black dot represents the Ryugu coordinates. HP reference frame.

\section{(10) UNIIERSITY PRESA}


Table 10 Nominal state vector at HRM and estimated states by Fujitsu (OPNAV1/NAV3 in Fig. 3), JAXA (OPNAV2/NAV3 in Fig. 3), and HPNAV (NAV1 in Fig. 3)

\begin{tabular}{cccccc}
\hline $\begin{array}{c}\text { State } \\
\text { estimate }\end{array}$ & $\begin{array}{c}x_{\mathrm{HP}} \\
(\mathrm{km})\end{array}$ & $\begin{array}{c}y_{\mathrm{HP}} \\
(\mathrm{km})\end{array}$ & $\begin{array}{c}z_{\mathrm{HP}} \\
(\mathrm{km})\end{array}$ & $\begin{array}{c}v_{x_{\mathrm{HP}}} \\
(\mathrm{mm} / \mathrm{s})\end{array}$ \\
\hline Nominal & -0.0993 & -0.0217 & 20.3407 & 0.7 & $\begin{array}{c}v_{z_{\mathrm{HP}}} \\
(\mathrm{mm} / \mathrm{s})\end{array}$ \\
OD's Fujitsu & -0.6307 & -0.0807 & 19.9176 & 3.3 & 0.1 \\
OD's JAXA & -0.5825 & -0.0923 & 19.7616 & 3.0 & 0.4 \\
HPNAV & -0.6572 & -0.0527 & 19.8053 & 0.5 & 3.4 \\
\hline \hline
\end{tabular}

corresponding to the estimate solutions are shown in Table 11. The $\Delta V \mathrm{~s}$ in Table 11 were cross evaluated with the different solutions in Table 10 and the solutions were propagated with goNEAR. From this analysis, the solution from Fujitsu was selected for the $\Delta V$ planning at HRM.

(b) Figure 24 shows the .way file prepared on 2018/12/30 for the combined HRM and HPK maneuvers (2018/12/31). The OD's Fujitsu team .way file was the input for the AOCS team and the $\Delta V$ was computed in a body-fixed frame.

(c) The HRM $\Delta V$ accounts for the brake velocity $\Delta V$ at HRM plus the HPK $\Delta V$ to target the center of BOX-A operation on 2018/12/31. A lower $\Delta V$ execution of $3 \mathrm{~mm} / \mathrm{s}$ in both the $X$ and $Y$ directions was experienced, which allowed braking velocity in the $Z$ direction but not the lateral direction, as

Table 11 Nominal $\Delta V$, OD's Fujitsu $\Delta V$ (OPNAV1/NAV3 in Fig. 3), and OD's JAXA $\Delta V$ (OPNAV2/NAV3 in Fig. 3)

\begin{tabular}{cccc}
\hline $\begin{array}{c}\text { State } \\
\text { estimate }\end{array}$ & $\begin{array}{c}\Delta V_{X_{\mathrm{HP}}} \\
(\mathrm{cm} / \mathrm{s})\end{array}$ & $\begin{array}{c}\Delta V_{Y_{\mathrm{HP}}} \\
(\mathrm{cm} / \mathrm{s})\end{array}$ & $\begin{array}{c}\Delta V_{Z_{\mathrm{HP}}} \\
(\mathrm{cm} / \mathrm{s})\end{array}$ \\
\hline Nominal & 0.2650 & -0.0610 & 1.3410 \\
OD's Fujitsu & 0.3090 & -0.0560 & 1.3750 \\
OD's JAXA & 0.3020 & -0.0550 & 1.3800 \\
\hline
\end{tabular}

shown in Fig. 25. Figure 25 shows the planned and actual predicted trajectory after the HRM maneuver.

The lower $\Delta V$ given is due to a decrease in pressure in the fuel tank, which required adjustment after the conjunction operation, as shown in Fig. 25. The error between the planned and the actual $\Delta V$ at HRM was $89.29 \%$ in $\Delta V_{X_{B}}, 92.15 \%$ in $\Delta V_{Y_{B}}$, and $97.43 \%$ in $\Delta V_{Z_{B}}$, as shown in the report of Fig. 26. Therefore, on 2018/12/31 a BOX-A operation was performed to bring the spacecraft back to $20 \mathrm{~km}$. 2018/12/29 marked the end of the superior solar conjunction phase and the start of the second half of the Ryugu proximity operations.

\subsection{Summary of the solar conjunction operation}

In this section, the results of the COI $(2018 / 11 / 23)$, TCM1 (2018/11/30), TCM2 (2018/12/25), and HRM (2018/12/29) operations are shown. The deterministic $\Delta V$ was computed for each trajectory leg, using the optNEAR tool. The comparison between the planned trajectory after the OD campaign and the actual $\Delta V$ trajectory after the mission operation are shown for COI (Fig. 10), TCM1 (Fig. 13), TCM2 (Fig. 23), and

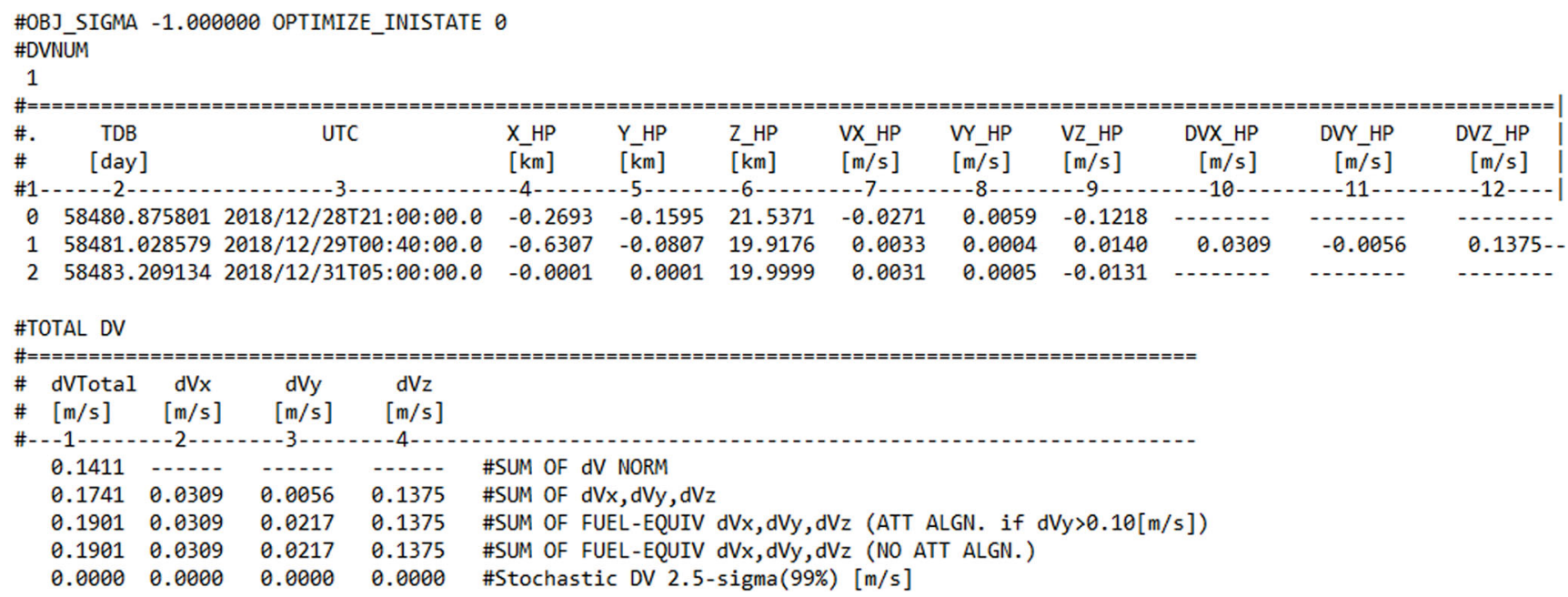

Fig. 24 Planned $\Delta V$ at HRM (including HPK) in .way file format. 


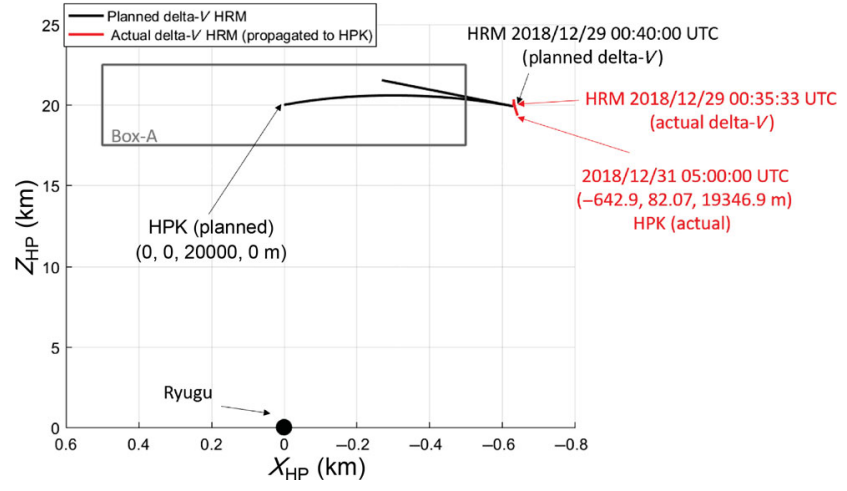

Fig. 25 Planned trajectory OD Fujitsu solution (black) and the actual trajectory after HRM operation (red). The edges of BOX-A are marked in gray. The black dot represents the Ryugu coordinates. HP reference frame.

\begin{tabular}{|c|c|c|c|c|c|}
\hline \multirow{2}{*}{ 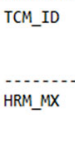 } & \multirow{2}{*}{$\begin{array}{c}\text { SC_TIME }_{\text {[UTC }} \\
00: 35: 33\end{array}$} & \multirow{2}{*}{$\begin{array}{l}\begin{array}{l}\text { Speed } \\
\text { Increase } \\
{[\mathrm{m} / \mathrm{s}]}\end{array} \\
0.0311 \\
0.0277\end{array}$} & \multirow{2}{*}{$\begin{array}{l}\text { Injection } \\
\text { length } \\
\text { [sec] }\end{array}$} & \multicolumn{2}{|l|}{ Remarks } \\
\hline & & & & \multicolumn{2}{|c|}{$\begin{array}{l}\text { Planned Value } \\
\text { ACM Integral Value }\end{array}$} \\
\hline HRM_MZ & $00: 40: 38$ & $\begin{array}{l}0.1000 \\
0.09337 \\
0.09308\end{array}$ & 1.503 & \multicolumn{2}{|c|}{$\begin{array}{l}\text { Planned Value (IIMEREQ, } 2 \text { injections) } \\
\text { Doppler Measuments } \\
\text { ACM Integral Value }\end{array}$} \\
\hline HRM_MY & $00: 45: 33$ & $\begin{array}{l}-0.0053 \\
-0.00488\end{array}$ & -0.319 & \multicolumn{2}{|c|}{$\begin{array}{l}\text { Planned Value } \\
\text { ACM Integral Value }\end{array}$} \\
\hline HRM_TZ & $02: 05: 33$ & $\begin{array}{l}0.0375 \\
0.0441 \\
0.0406 \\
0.0409\end{array}$ & $\begin{array}{l}0.575 \\
0.673\end{array}$ & \multicolumn{2}{|c|}{$\begin{array}{l}\text { Planned Value (Initially, } 2 \text { injections) } \\
\text { Planned Value (HRM_MZ Influence, } 2 \text { injections) } \\
\text { Doppler Measuments } \\
\text { ACM Integral Value }\end{array}$} \\
\hline Accuracy & $\begin{array}{l}\text { DVX } \\
\text { DVY } \\
\text { DVZ }\end{array}$ & \multicolumn{3}{|c|}{$\begin{array}{l}0.0277 / 0.0311 \\
0.00488 / \theta .0053 \\
(0.09337+0.0406) /(0.1000+0.0375)\end{array}$} & $\begin{array}{l}=89.29 \% \\
=92.15 \% \\
=97.43 \%\end{array}$ \\
\hline & $\begin{array}{l}\text { DVX } \\
\text { DVY } \\
\text { DVZ }\end{array}$ & $\begin{array}{l}0.0277-\theta . \\
\theta .00488-\theta \\
(\theta .09337\end{array}$ & $\begin{array}{l}0311 \\
.0053 \\
+0.0406)-(\theta\end{array}$ & $.1000+0.0375)$ & $\begin{array}{l}=-0.0034 \\
=-0.00042 \\
=-0.003530\end{array}$ \\
\hline
\end{tabular}

Fig. 26 Results of the HRM (including HPK) operation on 2018/12/29: planned and measured $\Delta V$ report.
HRM (Fig. 25). In Figs. 10, 13, 23, and 25, the trajectory in black is the planned trajectory, while the one in red is the propagated trajectory after measuring the actual $\Delta V$. The deterministic $\Delta V$ was computed using optNEAR in J2000EQ and then given in the HP and spacecraft's body-fixed reference frames, as shown in Table 12. As previously mentioned, $\Delta V$ slower than $1 \mathrm{~mm} / \mathrm{s}$ was neglected while $\Delta V$ faster than $10 \mathrm{~cm} / \mathrm{s}$ was executed two times as main and trim $\Delta V \mathrm{~s}$, as shown in Table 13. Table 13 shows the effects on the $\Delta V$ of truncation of up to 4 digits of the $\Delta V$ command. Evidently, both TCM1 and TCM2 did not require a trim $\Delta V$, while the $Z$ direction required main and trim $\Delta V \mathrm{~s}$ for both COI and HRM. The planned trim $\Delta V$ was usually rescheduled during the mission operation to compensate for the error of the main $\Delta V$. In Table 13, the actual measurements are the ACMs for the $X$ and $Y \Delta V$ s and 2-way Doppler for the $Z \Delta V$.

\section{Conclusions}

In this article, the Hayabusa2's low energy conjunction (ayu) trajectory, executed in late 2018, was presented. As a result of the operation, the optNEAR tool was validated in real time and was used for the validation of JAXA's JATOPS trajectory design tool at high altitude operations. The spacecraft reached a maximum distance of $109 \mathrm{~km}$ from Ryugu on 2018/12/11 and returned to home position (20 km from Ryugu) on 2018/12/29 after 21 days of uncontrolled orbital motion.

Table 12 Designed deterministic $\Delta V$ in HP and body-fixed frames

\begin{tabular}{lrrrrrr}
\hline Maneuver & $\Delta V_{X_{\mathrm{HP}}}$ & $\Delta V_{Y_{\mathrm{HP}}}$ & $\Delta V_{Z_{\mathrm{HP}}}$ & $\Delta V_{X_{B}}$ & $\Delta V_{Y_{B}}$ & $\Delta V_{Z_{B}}$ \\
\hline COI $(\mathrm{cm} / \mathrm{s})$ & 2.154758 & -0.290556 & 14.049689 & -2.071104 & 0.595609 & 14.052644 \\
TCM1 $(\mathrm{cm} / \mathrm{s})$ & 0.049176 & 0.004165 & 0.379261 & -0.048507 & 0.005917 & 0.379325 \\
TCM2 $(\mathrm{cm} / \mathrm{s})$ & -0.708705 & 0.263339 & -0.585655 & -0.719433 & 0.234432 & -0.584859 \\
$\mathrm{HRM}(\mathrm{cm} / \mathrm{s})$ & 3.092521 & -0.560379 & 13.753272 & 3.111279 & -0.530874 & 13.750199 \\
\hline
\end{tabular}

Table 13 Planned and actual executed $\Delta V$ in the body-fixed frame

\begin{tabular}{|c|c|c|c|c|c|c|}
\hline Maneuver & $\begin{array}{r}\Delta V_{X_{B}} \\
\text { (planned) }\end{array}$ & $\begin{array}{r}\Delta V_{Y_{B}} \\
\text { (planned) }\end{array}$ & $\begin{array}{r}\Delta V_{Z_{B}} \\
\text { (planned) }\end{array}$ & $\begin{array}{r}\Delta V_{X_{B}} \\
\text { (actual) }\end{array}$ & $\begin{array}{r}\Delta V_{Y_{B}} \\
\text { (actual) }\end{array}$ & $\begin{array}{r}\Delta V_{Z_{B}} \\
\text { (actual) }\end{array}$ \\
\hline COI (main) $(\mathrm{cm} / \mathrm{s})$ & -2.0700 & 0.5900 & 10.0000 & -2.0448 & 0.54918 & 9.4530 \\
\hline COI $($ trim $)(\mathrm{cm} / \mathrm{s})$ & 0.0000 & 0.0000 & 4.5970 & 0.0000 & 0.0000 & 4.3900 \\
\hline TCM1 (main) (cm/s) & 0.0000 & 0.0000 & 0.3800 & 0.0000 & 0.0000 & 0.3770 \\
\hline TCM1 $(\operatorname{trim})(\mathrm{cm} / \mathrm{s})$ & 0.0000 & 0.0000 & 0.0000 & 0.0000 & 0.0000 & 0.0000 \\
\hline TCM2 (main) $(\mathrm{cm} / \mathrm{s})$ & -0.7206 & 0.2310 & -0.5892 & -0.7631 & 0.2315 & -0.5508 \\
\hline TCM2 (trim) $(\mathrm{cm} / \mathrm{s})$ & 0.0000 & 0.0000 & 0.0000 & 0.0000 & 0.0000 & 0.0000 \\
\hline $\operatorname{HRM}($ main $)(\mathrm{cm} / \mathrm{s})$ & 3.0900 & -0.5600 & 10.0000 & 2.7700 & -0.4880 & 9.3370 \\
\hline $\operatorname{HRM}($ trim $)(\mathrm{cm} / \mathrm{s})$ & 0.0000 & 0.0000 & 4.4100 & 0.0000 & 0.0000 & 4.0600 \\
\hline
\end{tabular}


Due to the error in the attitude maintenance in deep conjunction, Ryugu was not in the FOV of the ONC-T camera on $2019 / 12 / 15$, while it was consistently in the FOV of ONC-W1. On 2018/12/11 (deep conjunction), a beacon operation was performed for radio science purposes to test the Ka-band capability under solar corona noise. The total expenditure in the $\Delta V$ was, as desired, less than $0.36 \mathrm{~m} / \mathrm{s}$. Based on the observation data collected during the solar conjunction, the orbit of Ryugu was recalculated. This updated orbit was used after returning to the HP position (HRM) to resume the home position at an altitude of $20 \mathrm{~km}$ above the asteroid surface. It was confirmed that using this new orbit of Ryugu achieves and maintains a more stable home position.

\section{Appendix A: Partial derivatives of the optNEAR's linearized equations}

The partial derivatives including the gravity of Ryugu, the 3rd body, and the SRB perturbations are given by the following equation:

$$
\begin{aligned}
& {\left[\begin{array}{lll}
\frac{\partial F_{4}}{\partial x} & \frac{\partial F_{4}}{\partial y} & \frac{\partial F_{4}}{\partial z} \\
\frac{\partial F_{5}}{\partial x} & \frac{\partial F_{5}}{\partial y} & \frac{\partial F_{5}}{\partial z} \\
\frac{\partial F_{6}}{\partial x} & \frac{\partial F_{6}}{\partial y} & \frac{\partial F_{6}}{\partial z}
\end{array}\right]=\left[\begin{array}{lll}
\frac{\partial F_{4 r}}{\partial x} & \frac{\partial F_{4 r}}{\partial y} & \frac{\partial F_{4 r}}{\partial z} \\
\frac{\partial F_{5 r}}{\partial x} & \frac{\partial F_{5 r}}{\partial y} & \frac{\partial F_{5 r}}{\partial z} \\
\frac{\partial F_{6 r}}{\partial x} & \frac{\partial F_{6 r}}{\partial y} & \frac{\partial F_{6 r}}{\partial z}
\end{array}\right]+} \\
& \sum_{j=1}^{N P_{j}}\left[\begin{array}{lll}
\frac{\partial F_{4 p_{j}}}{\partial x} & \frac{\partial F_{4 p_{j}}}{\partial y} & \frac{\partial F_{4 p_{j}}}{\partial z} \\
\frac{\partial F_{5 p_{j}}}{\partial x} & \frac{\partial F_{5 p_{j}}}{\partial y} & \frac{\partial F_{5 p_{j}}}{\partial z} \\
\frac{\partial F_{6 p_{j}}}{\partial x} & \frac{\partial F_{6 p_{j}}}{\partial y} & \frac{\partial F_{6 p_{j}}}{\partial z}
\end{array}\right]+\left[\begin{array}{lll}
\frac{\partial F_{4 \mathrm{SRP}}}{\partial x} & \frac{\partial F_{4 \mathrm{SRP}}}{\partial y} & \frac{\partial F_{4 \mathrm{SRP}}}{\partial z} \\
\frac{\partial F_{5 \mathrm{SRP}}}{\partial x} & \frac{\partial F_{5 \mathrm{SRP}}}{\partial y} & \frac{\partial F_{5 \mathrm{SRP}}}{\partial z} \\
\frac{\partial F_{6 \mathrm{SRP}}}{\partial x} & \frac{\partial F_{6 \mathrm{SRP}}}{\partial y} & \frac{\partial F_{6 \mathrm{SRP}}}{\partial z}
\end{array}\right]
\end{aligned}
$$

\section{A.1 Partial derivatives of the Ryugu's gravity}

The partial derivatives of Ryugu's gravity are given by

$$
\begin{aligned}
& {\left[\begin{array}{lll}
\frac{\partial F_{4 r}}{\partial x} & \frac{\partial F_{4 r}}{\partial y} & \frac{\partial F_{4 r}}{\partial z} \\
\frac{\partial F_{5 r}}{\partial x} & \frac{\partial F_{5 r}}{\partial y} & \frac{\partial F_{5 r}}{\partial z} \\
\frac{\partial F_{6 r}}{\partial x} & \frac{\partial F_{6 r}}{\partial y} & \frac{\partial F_{6 r}}{\partial z}
\end{array}\right]=} \\
& {\left[\begin{array}{ccc}
-\frac{\mu_{a}}{r^{3}}\left(1-\frac{3 x^{2}}{r^{2}}\right) & \mu_{a} \frac{3 x y}{r^{5}} & \mu_{a} \frac{3 x z}{r^{5}} \\
\mu_{a} \frac{3 x y}{r^{5}} & -\frac{\mu_{a}}{r^{3}}\left(1-\frac{3 y^{2}}{r^{2}}\right) & \mu_{a} \frac{3 y z}{r^{5}} \\
\mu_{a} \frac{3 x z}{r^{5}} & \mu_{a} \frac{3 y z}{r^{5}} & -\frac{\mu_{a}}{r^{3}}\left(1-\frac{3 z^{2}}{r^{2}}\right)
\end{array}\right]}
\end{aligned}
$$

\section{A.2 Partial derivatives of the 3rd body perturbations}

The partial derivatives of the 3rd body perturbation are derived as

$$
\begin{aligned}
& {\left[\begin{array}{ccc}
\frac{\partial F_{4 p_{j}}}{\partial x} & \frac{\partial F_{4 p_{j}}}{\partial y} & \frac{\partial F_{4 p_{j}}}{\partial z} \\
\frac{\partial F_{5 p_{j}}}{\partial x} & \frac{\partial F_{5 p_{j}}}{\partial y} & \frac{\partial F_{5 p_{j}}}{\partial z} \\
\frac{\partial F_{6 p_{j}}}{\partial x} & \frac{\partial F_{6 p_{j}}}{\partial y} & \frac{\partial F_{6 p_{j}}}{\partial z}
\end{array}\right]=\left[\begin{array}{c}
-\frac{\mu_{P j}}{\Delta^{3}}\left[1-\frac{3\left(x-d_{x}\right)^{2}}{\Delta^{2}}\right.
\end{array}\right]} \\
& \mu_{P j} \frac{3\left(x-d_{x}\right)\left(y-d_{y}\right)}{\Delta^{5}} \quad \mu_{P j} \frac{3\left(x-d_{x}\right)\left(z-d_{z}\right)}{\Delta^{5}} \\
& -\frac{\mu_{P j}}{\Delta^{3}}\left[1-\frac{3\left(y-d_{y}\right)^{2}}{\Delta^{2}}\right] \quad \mu_{P j} \frac{3\left(y-d_{z}\right)\left(z-d_{z}\right)}{\Delta^{5}} \\
& \mu_{P j} \frac{3\left(y-d_{y}\right)\left(z-d_{z}\right)}{\Delta^{5}} \quad-\frac{\mu_{P j}}{\Delta^{3}}\left[1-\frac{3\left(z-d_{z}\right)^{2}}{\Delta^{2}}\right]
\end{aligned}
$$

\section{A.3 Partial derivatives of solar radiation pressure perturbation}

The cannonball model assumes that the Hayabusa2 spacecraft is Sun-pointing. This is not exact during the conjunction phase when Hayabusa2 is kept Earthpointing. Therefore, the equations for a flat surface are more appropriate and used here. The partial derivatives of Eq. (4) are given by rearranging Eq. (4) as follows:

$$
\begin{aligned}
\boldsymbol{a}_{\mathrm{SRP}}= & -\frac{K(1-\epsilon)}{r_{\text {Earth }}} \frac{\boldsymbol{r}_{\mathrm{Earth}} \cdot \boldsymbol{r}_{\mathrm{ls}}}{r_{\mathrm{ls}}^{4}} \boldsymbol{r}_{\mathrm{ls}} \\
& -\frac{2 K \epsilon}{r_{\text {Earth }}^{3}} \frac{\left(\boldsymbol{r}_{\mathrm{Earth}} \cdot \boldsymbol{r}_{\mathrm{ls}}\right)^{2}}{r_{\mathrm{ls}}^{4}} \boldsymbol{r}_{\text {Earth }}
\end{aligned}
$$

with

$$
K=\frac{P_{0}}{c} \frac{A}{m} A U^{2}
$$

Note that:

$$
\boldsymbol{r}_{\text {Earth }}=\left\{\begin{array}{c}
X_{\mathrm{E}} \\
Y_{\mathrm{E}} \\
Z_{\mathrm{E}}
\end{array}\right\} \quad \text { and } \quad \boldsymbol{r}_{\mathrm{ls}}=\left\{\begin{array}{c}
X_{\mathrm{S}}-X \\
Y_{\mathrm{S}}-Y \\
Z_{\mathrm{S}}-Z
\end{array}\right\}
$$

The derivatives of the two terms in Eq. (24) are derived by components and it was distinguished between the 1st term of Eq. (24) and 2nd term of Eq. (24). The partial derivatives of SRP are

$\left[\begin{array}{lll}\frac{\partial F_{4 \mathrm{SRP}}}{\partial x} & \frac{\partial F_{4 \mathrm{SRP}}}{\partial y} & \frac{\partial F_{4 \mathrm{SRP}}}{\partial z} \\ \frac{\partial F_{5 \mathrm{SRP}}}{\partial x} & \frac{\partial F_{5 \mathrm{SRP}}}{\partial y} & \frac{\partial F_{5 \mathrm{SRP}}}{\partial z} \\ \frac{\partial F_{6 \mathrm{SRP}}}{\partial x} & \frac{\partial F_{6 \mathrm{SRP}}}{\partial y} & \frac{\partial F_{6 \mathrm{SRP}}}{\partial z}\end{array}\right]=-\frac{K(1-\epsilon)}{r_{\text {Earth }}}\left[\begin{array}{lll}a_{1 x x} & a_{1 x y} & a_{1 x z} \\ a_{1 y x} & a_{1 y y} & a_{1 y z} \\ a_{1 z x} & a_{1 z y} & a_{1 z z}\end{array}\right]$ 


$$
-\frac{2 K \epsilon}{r_{\text {Earth }}^{3}}\left[\begin{array}{lll}
a_{2 x x} & a_{2 x y} & a_{2 x z} \\
a_{2 y x} & a_{2 y y} & a_{2 y z} \\
a_{2 z x} & a_{2 z y} & a_{2 z z}
\end{array}\right]
$$

This formulation was written for flat surface Earthpointing, and therefore the norm is a function of the Earth-asteroid line, for simplicity. The two terms of Eq. (27) are derived by components as

1) $1 \mathrm{st}$ term

$$
\begin{aligned}
\frac{\boldsymbol{r}_{\mathrm{Earth}} \cdot \boldsymbol{r}_{\mathrm{ls}}}{r_{\mathrm{ls}}^{4}} \boldsymbol{r}_{\mathrm{ls}}= & \frac{X_{\mathrm{E}}\left(X_{\mathrm{S}}-X\right)+Y_{\mathrm{E}}\left(Y_{\mathrm{S}}-Y\right)+Z_{\mathrm{E}}\left(Z_{\mathrm{S}}-Z\right)}{\left[\left(X_{\mathrm{S}}-X\right)^{2}+\left(Y_{\mathrm{S}}-Y\right)^{2}+\left(Z_{\mathrm{S}}-Z\right)^{2}\right]^{2}} . \\
& \left\{\begin{array}{c}
X_{\mathrm{S}}-X \\
Y_{\mathrm{S}}-Y \\
Z_{\mathrm{S}}-Z
\end{array}\right\}=\boldsymbol{a}_{1}
\end{aligned}
$$

Derivatives of the $x$-component

$$
\begin{aligned}
\frac{\partial a_{1 x}}{\partial x}= & a_{1 x x}=-\frac{2 X_{\mathrm{E}}\left(X_{\mathrm{S}}-X\right)+Y_{\mathrm{E}}\left(Y_{\mathrm{S}}-Y\right)+Z_{\mathrm{E}}\left(Z_{\mathrm{S}}-Z\right)}{r_{\mathrm{ls}}^{4}}+ \\
& 4 \frac{\boldsymbol{r}_{\mathrm{Earth}} \cdot \boldsymbol{r}_{\mathrm{ls}}\left(X_{\mathrm{S}}-X\right)^{2}}{r_{\mathrm{ls}}^{6}} \\
\frac{\partial a_{1 x}}{\partial y}= & a_{1 x y}=-\frac{Y_{\mathrm{E}}\left(X_{\mathrm{S}}-X\right)}{r_{\mathrm{ls}}^{4}}+ \\
& 4 \frac{\boldsymbol{r}_{\mathrm{Earth}} \cdot \boldsymbol{r}_{\mathrm{ls}}\left(X_{\mathrm{S}}-X\right)\left(Y_{\mathrm{S}}-Y\right)}{r_{\mathrm{ls}}^{6}} \\
\frac{\partial a_{1 x}}{\partial z}= & a_{1 x z}=-\frac{Z_{\mathrm{E}}\left(X_{\mathrm{S}}-X\right)}{r_{\mathrm{ls}}^{4}}+ \\
& 4 \frac{\boldsymbol{r}_{\mathrm{Earth}} \cdot \boldsymbol{r}_{\mathrm{ls}}\left(X_{\mathrm{S}}-X\right)\left(Z_{\mathrm{S}}-Z\right)}{r_{\mathrm{ls}}^{6}}
\end{aligned}
$$

Derivative of the $y$-component

$$
\begin{aligned}
\frac{\partial a_{1 y}}{\partial x}= & a_{1 y x}=-\frac{X_{\mathrm{E}}\left(Y_{\mathrm{S}}-Y\right)}{r_{\mathrm{ls}}^{4}}+ \\
& 4 \frac{\boldsymbol{r}_{\mathrm{Earth}} \cdot \boldsymbol{r}_{\mathrm{ls}}\left(Y_{\mathrm{S}}-Y\right)\left(X_{\mathrm{S}}-X\right)}{r_{\mathrm{ls}}^{6}}
\end{aligned}
$$

$$
\begin{aligned}
\frac{\partial a_{1 y}}{\partial y}= & a_{1 y y}=-\frac{X_{\mathrm{E}}\left(X_{\mathrm{S}}-X\right)+2 Y_{\mathrm{E}}\left(Y_{\mathrm{S}}-Y\right)+Z_{\mathrm{E}}\left(Z_{\mathrm{S}}-Z\right)}{r_{\mathrm{ls}}^{4}}+ \\
& 4 \frac{\boldsymbol{r}_{\mathrm{Earth}} \cdot \boldsymbol{r}_{\mathrm{ls}}\left(Y_{\mathrm{S}}-Y\right)^{2}}{r_{\mathrm{ls}}^{6}}
\end{aligned}
$$

$$
\begin{aligned}
\frac{\partial a_{1 y}}{\partial z}= & a_{1 y z}=-\frac{Z_{\mathrm{E}}\left(Y_{\mathrm{S}}-Y\right)}{r_{\mathrm{ls}}^{4}}+ \\
& 4 \frac{\boldsymbol{r}_{\mathrm{Earth}} \cdot \boldsymbol{r}_{\mathrm{ls}}\left(Y_{\mathrm{S}}-Y\right)\left(Z_{\mathrm{S}}-Z\right)}{r_{\mathrm{ls}}^{6}}
\end{aligned}
$$

Derivative of the $z$-component

$$
\begin{aligned}
\frac{\partial a_{1 z}}{\partial x}= & a_{1 z x}=-\frac{X_{\mathrm{E}}\left(Z_{\mathrm{S}}-Z\right)}{r_{\mathrm{ls}}^{4}}+ \\
& 4 \frac{\boldsymbol{r}_{\mathrm{Earth}} \cdot \boldsymbol{r}_{\mathrm{ls}}\left(Z_{\mathrm{S}}-Z\right)\left(X_{\mathrm{S}}-X\right)}{r_{\mathrm{ls}}^{6}}
\end{aligned}
$$

$$
\begin{aligned}
\frac{\partial a_{1 z}}{\partial y}= & a_{1 z y}=-\frac{Y_{\mathrm{E}}\left(Z_{\mathrm{S}}-Z\right)}{r_{\mathrm{ls}}^{4}}+ \\
& 4 \frac{\boldsymbol{r}_{\mathrm{Earth}} \cdot \boldsymbol{r}_{\mathrm{ls}}\left(Z_{\mathrm{S}}-Z\right)\left(Y_{\mathrm{S}}-Y\right)}{r_{\mathrm{ls}}^{6}} \\
\frac{\partial a_{1 z}}{\partial z}= & a_{1 z z}=-\frac{X_{\mathrm{E}}\left(X_{\mathrm{S}}-X\right)+Y_{\mathrm{E}}\left(Y_{\mathrm{S}}-Y\right)+2 Z_{\mathrm{E}}\left(Z_{\mathrm{S}}-Z\right)}{r_{\mathrm{ls}}^{4}}+ \\
& 4 \frac{\boldsymbol{r}_{\mathrm{Earth}} \cdot \boldsymbol{r}_{\mathrm{ls}}\left(Z_{\mathrm{S}}-Z\right)^{2}}{r_{\mathrm{ls}}^{6}}
\end{aligned}
$$

2) 2nd term

$$
\begin{aligned}
& \frac{\left(\boldsymbol{r}_{\mathrm{Earth}} \cdot \boldsymbol{r}_{\mathrm{ls}}\right)^{2}}{r_{\mathrm{ls}}^{4}} \boldsymbol{r}_{\mathrm{Earth}}= \\
& \frac{\left[X_{\mathrm{E}}\left(X_{\mathrm{S}}-X\right)+Y_{\mathrm{E}}\left(Y_{\mathrm{S}}-Y\right)+Z_{\mathrm{E}}\left(Z_{\mathrm{S}}-Z\right)\right]^{2}}{\left[\left(X_{\mathrm{S}}-X\right)^{2}+\left(Y_{\mathrm{S}}-Y\right)^{2}+\left(Z_{\mathrm{S}}-Z\right)^{2}\right]^{2}}\left\{\begin{array}{c}
X_{\mathrm{E}} \\
Y_{\mathrm{E}} \\
Z_{\mathrm{E}}
\end{array}\right\}=\boldsymbol{a}_{2}
\end{aligned}
$$

Partial derivatives of

$$
\begin{aligned}
\frac{\partial}{\partial \boldsymbol{r}} \frac{\left(\boldsymbol{r}_{\mathrm{Earth}} \cdot \boldsymbol{r}_{\mathrm{ls}}\right)^{2}}{r_{\mathrm{ls}}^{4}}= & 2 \frac{\boldsymbol{r}_{\mathrm{Earth}} \cdot \boldsymbol{r}_{\mathrm{ls}}}{r_{\mathrm{ls}}^{4}} \frac{\partial\left(\boldsymbol{r}_{\mathrm{Earth}} \cdot \boldsymbol{r}_{\mathrm{ls}}\right)}{\partial \boldsymbol{r}}- \\
& \frac{\left(\boldsymbol{r}_{\mathrm{Earth}} \cdot \boldsymbol{r}_{\mathrm{ls}}\right)^{2}}{r_{\mathrm{ls}}^{8}} \frac{\partial r_{\mathrm{ls}}^{4}}{\partial \boldsymbol{r}}
\end{aligned}
$$

where

$$
\frac{\partial\left(\boldsymbol{r}_{\mathrm{Earth}} \cdot \boldsymbol{r}_{\mathrm{ls}}\right)}{\partial \boldsymbol{r}}=-\left\{\begin{array}{c}
X_{\mathrm{E}} \\
Y_{\mathrm{E}} \\
Z_{\mathrm{E}}
\end{array}\right\}
$$

and

$$
\frac{\partial r_{\mathrm{ls}}^{4}}{\partial \boldsymbol{r}}=-4\left[\left(X_{\mathrm{S}}-X\right)^{2}+\left(Y_{\mathrm{S}}-Y\right)^{2}+\left(Z_{\mathrm{S}}-Z\right)^{2}\right]\left\{\begin{array}{c}
X_{\mathrm{S}}-X \\
Y_{\mathrm{S}}-Y \\
Z_{\mathrm{S}}-Z
\end{array}\right\}
$$


therefore

$$
\begin{aligned}
\frac{\partial}{\partial \boldsymbol{r}} \frac{\left(\boldsymbol{r}_{\mathrm{Earth}} \cdot \boldsymbol{r}_{\mathrm{ls}}\right)^{2}}{r_{\mathrm{ls}}^{4}}=-2 \frac{\boldsymbol{r}_{\mathrm{Earth}} \cdot \boldsymbol{r}_{\mathrm{ls}}}{r_{\mathrm{ls}}^{4}}\left\{\begin{array}{c}
X_{\mathrm{E}} \\
Y_{\mathrm{E}} \\
Z_{\mathrm{E}}
\end{array}\right\}+ \\
4 \frac{\left(\boldsymbol{r}_{\mathrm{Earth}} \cdot \boldsymbol{r}_{\mathrm{ls}}\right)^{2}}{r_{\mathrm{ls}}^{6}}\left\{\begin{array}{c}
X_{\mathrm{S}}-X \\
Y_{\mathrm{S}}-Y \\
Z_{\mathrm{S}}-Z
\end{array}\right\}
\end{aligned}
$$

Finally

$$
\begin{aligned}
{\left[\begin{array}{ccc}
a_{2 x x} & a_{2 x y} & a_{2 x z} \\
a_{2 y x} & a_{2 y y} & a_{2 y z} \\
a_{2 z x} & a_{2 z y} & a_{2 z z}
\end{array}\right]=} & {\left[-2 \frac{\boldsymbol{r}_{\mathrm{Earth}} \cdot \boldsymbol{r}_{\mathrm{ls}}}{r_{\mathrm{ls}}^{4}}\left\{\begin{array}{c}
X_{\mathrm{E}} \\
Y_{\mathrm{E}} \\
Z_{\mathrm{E}}
\end{array}\right\}+\right.} \\
& \left.4 \frac{\left(\boldsymbol{r}_{\mathrm{Earth}} \cdot \boldsymbol{r}_{\mathrm{ls}}\right)^{2}}{r_{\mathrm{ls}}^{6}}\left\{\begin{array}{c}
X_{\mathrm{S}}-X \\
Y_{\mathrm{S}}-Y \\
Z_{\mathrm{S}}-Z
\end{array}\right\}\right] \\
& {\left[\begin{array}{ccc}
X_{\mathrm{E}} & Y_{\mathrm{E}} & Z_{\mathrm{E}}
\end{array}\right] }
\end{aligned}
$$

\section{A.4 Partial derivatives of solar radiation pressure perturbation (Sun-pointing)}

For a cannonball model, the partial derivatives of the SRP perturbation are quite simple and they are given by

$$
\begin{aligned}
& {\left[\begin{array}{lll}
\frac{\partial F_{4 \mathrm{SRP}}}{\partial x} & \frac{\partial F_{4 \mathrm{SRP}}}{\partial y} & \frac{\partial F_{4 \mathrm{SRP}}}{\partial z} \\
\frac{\partial F_{5 \mathrm{SRP}}}{\partial x} & \frac{\partial F_{5 \mathrm{SRP}}}{\partial y} & \frac{\partial F_{5 \mathrm{SRP}}}{\partial z} \\
\frac{\partial F_{6 \mathrm{SRP}}}{\partial x} & \frac{\partial F_{6 \mathrm{SRP}}}{\partial y} & \frac{\partial F_{6 \mathrm{SRP}}}{\partial z}
\end{array}\right]=} \\
& {\left[\begin{array}{ccc}
\frac{K}{r^{3}}\left(1-\frac{3 x^{2}}{r^{2}}\right) & -K \frac{3 x y}{r^{5}} & -K \frac{3 x z}{r^{5}} \\
-K \frac{3 x y}{r^{5}} & \frac{K}{r^{3}}\left(1-\frac{3 y^{2}}{r^{2}}\right) & -K \frac{3 y z}{r^{5}} \\
-K \frac{3 x z}{r^{5}} & -K \frac{3 y z}{r^{5}} & \frac{K}{r^{3}}\left(1-\frac{3 z^{2}}{r^{2}}\right)
\end{array}\right]}
\end{aligned}
$$

with

$$
K=\frac{P_{0} C_{r}}{c} \frac{A_{s c}}{m_{s c}} A U^{2}
$$

\section{References}

[1] Watanabe, S., Hirabayashi, M., Hirata, N., Noguchi, R., Shimaki, Y., Ikeda, H., Tatsumi, E., Yoshikawa, M., Kikuchi, S., Yabuta, H., et al. Hayabusa2 observations of the top-shape carbonaceous asteroid 162173 Ryugu. Science, 2019, 364(6437): 268-272.
[2] Lauretta, D., Balram-Knutson, S., Beshore, E., Boynton, W., dAubigny, C., DellaG-iustina, D., Enos, H., Gholish, D., Hergenrother, C., Howell, E., et al. Osiris-REx: Sample return from asteroid (101955) bennu. Space Science Reviews, 2017, 212: 925-984.

[3] Tsuda, Y., Yoshikawa, M., Abe, M., Minamino, H., Nakazawa, S. System design of the Hayabusa2 - Asteroid sample return mission to 1999 JU3. Acta Astronautica, 2013, 91: 356-362.

[4] Morley, T., Budnik, F. Effects on spacecraft radiometric data at superior solar conjunction. In: Proceedings of the 20th International Symposium on Space Flight Dynamics, 2007: 24-28

[5] Yoshikawa, M., Yano, H., Kawaguchi, J., Fujiwara, A., Abe, M., Iwata, T., Tanaka, S., Mori, O., Yoshimitsu, T., Takagi, Y., et al. Technologies for future asteroid exploration: What we learned from Hayabusa mission. In: Proceedings of Spacecraft Reconnaissance of Asteroid and Comet Interiors, 2006: 3038.

[6] Soldini, S., Yamaguchi, T., Tsuda, Y., Saiki, T., Nakazawa, S. Hayabusa2's superior solar conjunction phase: Trajectory design, guidance and navigation. Space Science Reviews, 2020, https://doi.org/10.1007/s112-14020-00371-5.

[7] Soldini, S., Yamaguchi, T., Tsuda, Y., Saiki, T. Hayabusa2 mission solar conjunction trajectory for hovering satellite: Design, navigation and post-operation evaluation. In: Proceedings of the 29th AAS/AIAA Space Flight Mechanics Meeting, 2019: AAS 19-241.

[8] Uesugi, K., Matsuo, H., Kawaguchi, J., Hayashi, T. Japanese first double lunar swingby mission "Hiten". Acta Astronautica, 1991, 25(7): 347-355.

[9] Belbruno, E., Miller, A. A ballistic lunar capture trajectory for the Japanese spacecraft Hiten. Jet Propulsion Laboratory IOM 312/90.41371-EAB, 1990.

[10] Koon, W. S., Lo, M. W., Marsden, J. E., Ross, S. D. Dynamical systems, the three-body problem and space mission design. Equadiff 99, 2000: 1167-1181.

[11] Tsuda, Y., Takeuchi H., Ogawa, N. O. G., Kikuchi, S., Oki, Y., Ishiguro, M., Kuroda, D., Urakawa S., O. S., Hayabusa2 project team. Rendezvous to asteroid with highly uncertain ephemeris: Hayabusa2's ryuguapproach operation result. Astrodynamics, 2020, 4: 137147.

[12] Tsuda, Y., Ono, G., Saiki, T., Mimasu, Y., Ogawa, N., Terui, F. Solar radiation pressure-assisted fuel-free Sun tracking and its application to Hayabusa2. Journal of Spacecraft and Rockets, 2017, 54(6): 1284-1293.

[13] Ono, G., Tsuda, Y., Akatsuka, K., Saiki, T., Mimasu, Y., Ogawa, N., Terui, F. Generalized attitude model for momentum-biased solar sail spacecraft. Journal of Guidance, Control, and Dynamics, 2016, 39(7): 14911500 
[14] Tsuda, Y., Saiki, T., Funase, R., Mimasu, Y. Generalized attitude model for spinning solar sail spacecraft. Journal of Guidance, Control, and Dynamics, 2013, 36(4): 967-974.

[15] Takeuchi, H., Yoshikawa, K., Tokei, Y., Oki, Y., Kikuchi, S., Ikeda, H., Soldini, S., Ogawa, N., Mimasu, Y., Ono, G., et al. The deep-space multi-object orbit determination system and its application to Hayabusa2's asteroid proximity operations. Astrodynamics, 2020 , 4(4): 377-392.

[16] Soldini, S., Takanao, S., Ikeda, H., Wada, K., Yuichi, T., Hirata, N., Hirata, N. A generalised methodology for analytic construction of 1:1 resonances around irregular bodies: Application to the asteroid Ryugu's ejecta dynamics. Planetary and Space Science, 2020, 180: 104740

[17] Montenbruck, O., Gill, E. Satellite Orbits Model, Methods and Applications. New York: Springer-Verlag Berlin Heidelberg, 2000.

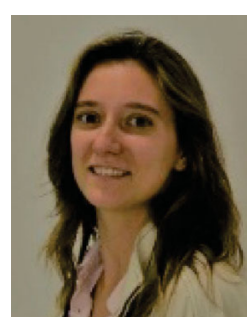

Stefania Soldini received her Ph.D. degree in October 2016 from the University of Southampton, UK. She is an assistant professor in space engineering in the University of Liverpool, UK. Since 2020, she has been the PI of the "3D printed self-folding origami solar sail" project funded by the CEII/EPSRC Network+ (https://www.youtube.com/ watch?v=U5IhFIxZxZI\&t=6s) in collaboration with Japan Aerospace Exploration Agency (JAXA) and Oxford Space Systems, UK. She is also a member of the Hayabusa2 Joint Science Team (HJST) as astrodynamics Co-I and member of the ESA's HERA and NASA's DART group. She was the PI of the "Hayabusa2" superior solar conjunction mission phase in late 2018. She worked at the JAXA's Institute of Space and Astronautical Science (ISAS) from 2016 to 2019, after completing one-year JSPS post-doc research fellowship at the same institute. Her research interests are astrodynamics, guidance, navigation \& control (GNC) for asteroids proximity operations, ejecta particles dynamics, planetary defence, solar sail technology, additive manufacturing, and AI. E-mail: stefania.soldini@liverpool.ac.uk.

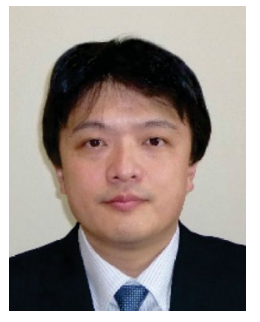

Hiroshi Takeuchi received his Ph.D. degree of science (physics and applied physics) from Waseda University in 2000. In 2006, he started working at ISAS/JAXA as a member of deep space orbit determination group. He was a visiting researcher of the NASA Jet Propulsion Laboratory in 2012-2013. He is currently an associate professor of ISAS/JAXA and is also the lead of the orbit determination of Hayabusa2. His current research interest is developing the deep space multi-objects orbit determination system. E-mail: takeuchi@jaxa.jp.

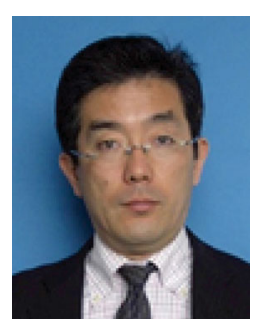

Sho Taniguchi completed his master's course in mechanical engineering at Ibaraki University in 1995. He joined Fujitsu in 1999, and engaged in tracking and tracking services at JAXA in 2005. In 2009, he was engaged in deep space orbit determination work at the Science System Solution Division of the TC Solution Business Unit, Fujitsu Limited. He is also a regular member of the Japanese Society of Astronautics. E-mail: taniguchi.sho@jp.fujitsu.com.

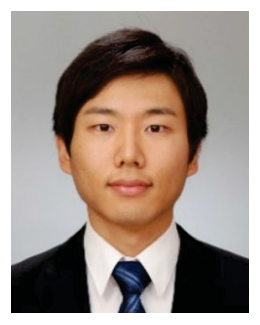

Shota Kikuchi received his Ph.D. degree in aeronautics and astronautics from the University of Tokyo in 2018. From 2015 to 2017, he had served as a visiting scholar at Purdue University and NASA Jet Propulsion Laboratory. He is currently a postdoctoral research associate at JAXA and is engaged in the Hayabusa2 asteroid sample-return mission as a system engineer. His primary research interests lie in the field of astrodynamics, particularly in dynamics around small bodies. E-mail: kikuchi.shota@jaxa.jp.

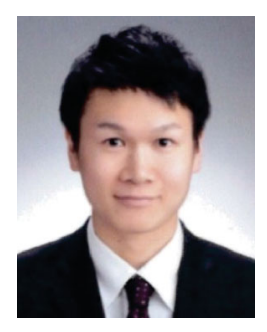

Yuto Takei received his Ph.D. degree in engineering from Tokyo Institute of Technology, Japan in 2015. He is a researcher at the Research and Development Directorate and JAXA. He is involved in the Hayabusa2 project as a systems engineer. His research interests include astrodynamics, spacecraft system, space robotics, and deep space exploration. E-mail: takei.yuto@jaxa.jp.

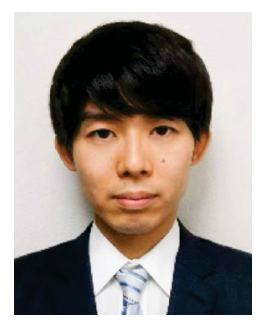

Go Ono is a researcher at JAXA. He graduated with his master degree in engineering from the University of Bath in 2011 and with his Ph.D. degree in aerospace engineering from the University of Tokyo in 2014. He joined JAXA in 2015 and has been working on guidance, navigation, and control systems of JAXA's deep space missions such as Hayabusa2 and MMX. His current research interests are astrodynamics and deep space exploration. E-mail: ono.go@jaxa.jp. 


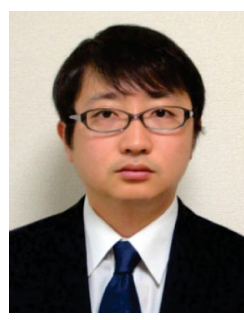

Takanao Saiki received his Ph.D. degree in aeronautics and astronautics from the University of Tokyo, Japan in 2005. He is an assistant professor at ISAS, JAXA. $\mathrm{He}$ is now involved in the Hayabusa2 project. He was a chief developer of the impact system and he is currently a project engineer. His research interests include astrodynamics, spacecraft system, and deep space exploration. Email: saiki.takanao@jaxa.jp.

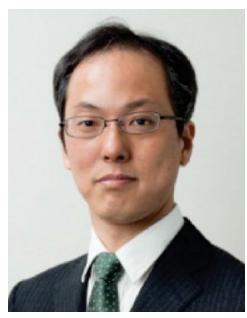

Yuichi Tsuda received his Ph.D. degree in aeronautics and astronautics from University of Tokyo in 2003 and joined JAXA in 2003 as a research associate. He had been a visiting scholar of the Department of Aerospace Engineering, University of Michigan and Department of Aerospace Engineering Sciences, University of Colorado Boulder in 2008-2009. He was a deputy lead of the IKAROS project, the world's first interplanetary solar sail mission. He is currently a professor of ISAS/JAXA and is the project manager of Hayabusa2, an asteroid sample-return mission. His research interests are astrodynamics, spacecraft system, and deep space exploration. E-mail: tsuda.yuichi@jaxa.jp.

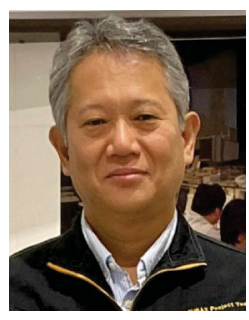

Fuyuto Terui received his Ph.D. degree in aerospace engineering from University of Osaka Prefecture in 1989. He has been a staff member of Space Technology Research Center of National Aerospace Laboratory (NAL) of Japan since 1989. He had been a visiting scholar of the University of Cambridge, Engineering Department, Control Group between 1994 and 1995. After the reorganization of space agencies in Japan, he has been a staff member of JAXA since 2003 and is now a function manager of the Hayabusa2 project as well as a representative of attitude and orbit control system of Hayabusa2 spacecraft. His main research fields include robust control and image-based guidance, and navigation and control of a spacecraft such as debris removal space robot and the asteroid exploration probe. E-mail: terui.fuyuto@jaxa.jp.

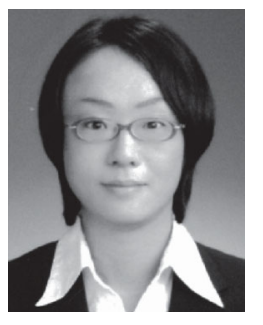

Naoko Ogawa received her B.E., M.E. and Ph.D. degrees in mathematical engineering and information physics in 2000, 2002 and 2005, respectively, from the University of Tokyo, Japan. From 2004 to 2008 she has been a research fellow of the Japan Society for the Promotion of Science. Since 2008, she has been a research engineer at ISAS and JAXA. Her current research interests include astrodynamics, mission design, robotics, spacecraft systems and operation. E-mail: naoko.ogawa@isas.jaxa.jp.

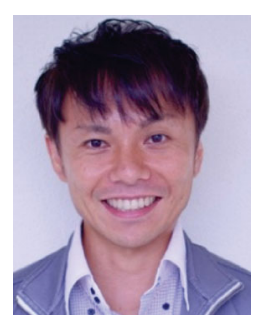

Yuya Mimasu is a researcher at JAXA. He graduated with his Ph.D. degree in aerospace engineering from Kyushu University. After graduation, he joined JAXA and has been working on the guidance, navigation, and control subsystem of Hayabusa2, which is JAXA's asteroid sample-return mission. His research interests are astrodynamics and mission analysis around small bodies. Email: mimasu.yuya@jaxa.jp.

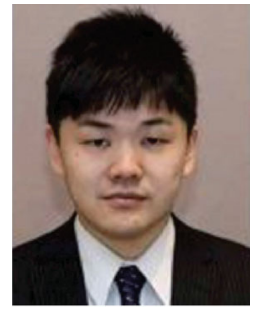

Atsushi Fujii is a researcher at Hayabusa2 Project Team, ISAS, JAXA. He received his bachelor of engineering from Saitama University, Japan. He involves in Hayabusa2 project as a ground system engineer.

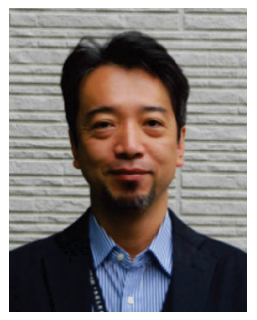

Satoru Nakazawa received his Ph.D. degree in science from Nagoya University, Japan in 1999. He is an assistant professor at ISAS and JAXA. He is now involved in the Hayabusa2 project. He is the deputy manager of the project team. His research interests include planetary science, spacecraft system engineering, and deep space exploration. Email: nakazawa.satoru@jaxa.jp.

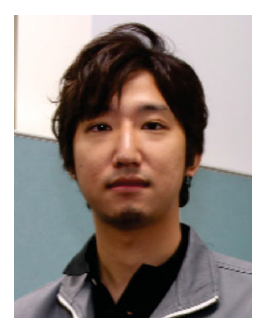

Kent Yoshikawa received his bachelor's and master's degrees in engineering from Tokyo Institute of Technology in 2013 and 2015, respectively. From 2015, he started working as an engineer in the Research and Development Directorate, JAXA. His current research interests include astrodynamics, GNC, planetary robotics, and planetary exploration. Email: yoshikawa.kento@jaxa.jp.

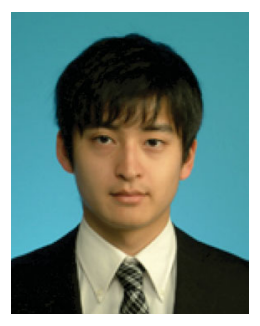

Yusuke Oki is a researcher at JAXA. He graduated with a master degree in astronautics from the University of Tokyo in 2016, and with a Ph.D. degree in astronautics from the University of Tokyo in 2019. He joined JAXA in 2019, and has been working on system design and orbit design of spacecrafts. His current research interests are astrodynamics, concurrent design, and deep space exploration. E-mail: oki.yusuke@jaxa.jp. 


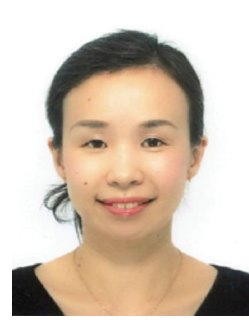

Chikako Hirose has worked at JAXA since 2004. She is currently a senior engineer and has had involvement in over 20 missions in flight dynamics field. She joined the Hayabusa2 team since 2018 after she finished her research at NASA as a visiting researcher for one year. Prior to that, she demonstrated her leadership in Venus orbit insertion of the Japanese first planet orbiter, Akatsuki. She obtained her master degree in physics from Ochanomizu University in 2004. E-mail: hirose.chikako@jaxa.jp.

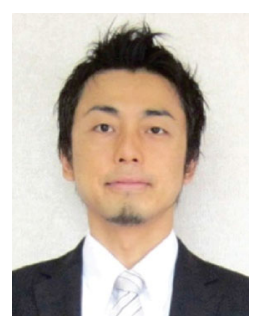

Hirotaka Sawada received his B.E. and M.E. degrees from the Tokyo Institute of Technology, Japan in 1998 and 2001, respectively. He received his Ph.D. degree from the Tokyo Institute of Technology, Japan in 2004. He is an associate senior engineer of JAXA. He is currently involved in the Martian Moons Exploration (MMX) project. His specialized field of research includes space robotics, dynamics, and control of space systems. E-mail: sawada.hirotaka@jaxa.jp.

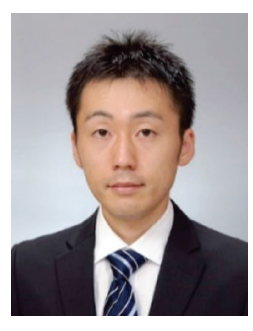

Tomohiro Yamaguchi is a system engineer of Mitsubishi Electric Corporation. He received his Ph.D. degree from the Graduate University for Advanced Studies, Japan in 2012. His career includes system design, mission analysis, and operations for interplanetary spacecrafts in both agencies and industry. His current research interests are system design and guidance, navigation and control for interplanetary spacecraft. E-mail: Yamaguchi.Tomohiro@ce.MitsubishiElectric.co.jp.

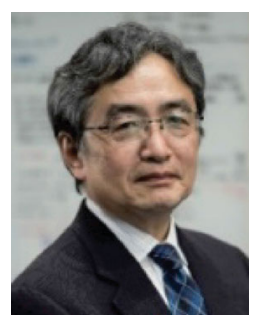

Makoto Yoshikawa is an associate professor in ISAS, JAXA. He is the mission manager of Hayabusa2 project. He got his Ph.D. degree in astronomy from the University of Tokyo in 1989. After working as a researcher of JSPS (Japan Society for the Promotion of Science), he worked at former Communication Research Laboratory from 1991 as senior researcher. He joined ISAS as associate professor in 1998. His research field is celestial mechanics, and he was involved in many space missions such as GEOTAIL, HALCA, Nozomi, Hayabusa, Akatsuki, and IKAROS. He is now also working for planetary defense issues. E-mail: yoshikawa.makoto@jaxa.jp.

Open Access This article is licensed under a Creative Commons Attribution 4.0 International License, which permits use, sharing, adaptation, distribution and reproduction in any medium or format, as long as you give appropriate credit to the original author(s) and the source, provide a link to the Creative Commons licence, and indicate if changes were made.

The images or other third party material in this article are included in the article's Creative Commons licence, unless indicated otherwise in a credit line to the material. If material is not included in the article's Creative Commons licence and your intended use is not permitted by statutory regulation or exceeds the permitted use, you will need to obtain permission directly from the copyright holder.

To view a copy of this licence, visit http://creativecommons.org/licenses/by/4.0/. 